\title{
WestVirginiaUniversity
}

THE RESEARCH REPOSITORY @ WVU

Graduate Theses, Dissertations, and Problem Reports

2004

\section{Graph minor}

Jianbing Niu

West Virginia University

Follow this and additional works at: https://researchrepository.wvu.edu/etd

\section{Recommended Citation}

Niu, Jianbing, "Graph minor" (2004). Graduate Theses, Dissertations, and Problem Reports. 2122.

https://researchrepository.wvu.edu/etd/2122

This Dissertation is protected by copyright and/or related rights. It has been brought to you by the The Research Repository @ WVU with permission from the rights-holder(s). You are free to use this Dissertation in any way that is permitted by the copyright and related rights legislation that applies to your use. For other uses you must obtain permission from the rights-holder(s) directly, unless additional rights are indicated by a Creative Commons license in the record and/ or on the work itself. This Dissertation has been accepted for inclusion in WVU Graduate Theses, Dissertations, and Problem Reports collection by an authorized administrator of The Research Repository @ WVU.

For more information, please contact researchrepository@mail.wvu.edu. 


\title{
Graph Minor
}

\author{
Jianbing Niu \\ Dissertation submitted to the \\ Eberly College of Arts and Sciences \\ at West Virginia University \\ in partial fulfillment of the requirements \\ for the degree of
}

Doctor of Philosophy

in

Mathematics
Cun-Quan Zhang, Ph.D., Chair
Elaine M. Eschen, Ph.D.
Hong-Jian Lai, Ph.D.
John L Goldwasser, Ph.D.
Jerzy Wojciechowski, Ph.D.

Department of Mathematics

\author{
Morgantown, West Virginia \\ 2004
}

Keywords: graph minor, graph embedding, connectivity Copyright 2004 Jianbing Niu 


\title{
ABSTRACT
}

\author{
Graph Minor
}

\section{Jianbing Niu}

In this paper, we present three results: (1) Let $G$ be a $(k+2)$-connected non-( $k-3)$-apex graph where $k \geq 2$. If $G$ contains three $k$-cliques, say $L_{1}, L_{2}, L_{3}$, such that $\left|L_{i} \cap L_{j}\right| \leq k-2$ $(1 \leq i<j \leq 3)$, then $G$ contains a $K_{k+2}$ as a minor. (2) Let $G$ be a 6 -connected claw-free graph. If $\delta(G) \geq 7$ and $G$ contains three disjoint 5 -cliques, say $L_{1}, L_{2}, L_{3}$, then $G$ contains a $K_{7}$ as a minor. (3) There is a function $h: N \longrightarrow N$, such that, for every 4-connected graph $G$ with minimum degree at least five embedded in a surface with Euler genus $g$ and face-width at least $h(g)$, every longest circuit of the graph $G$ has a chord. 


\section{Acknowledgments}

I would like to thank my supervisor, Dr. Cun-Quan Zhang, for his guidance, advice and continual encouragement. It is also my pleasure to work under his supervision. Without him, this dissertation could not have been completed.

I would also like to thank Dr. Hong-Jian Lai and other committee members: Dr. Elaine M. Eschen, Dr. John L Goldwasser, Dr. Jerzy Wojciechowski for their help during my studies. 


\section{DEDICATION}

To

my father, my mother 


\section{Contents}

1 Introduction $\quad 1$

2 Cliques, Minors and Apex Graphs $\quad 3$

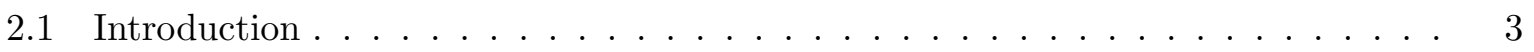

2.2 Terminology and notations $\ldots \ldots \ldots \ldots \ldots \ldots \ldots$

2.3 Lemmas . . . . . . . . . . . . . . . . . . . . . . 8

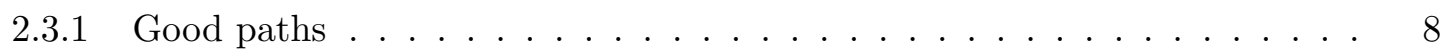

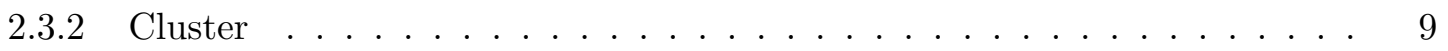

2.3 .3 The 6 -cluster lemma . . . . . . . . . . . . . . . . . . . 10

2.4 Proof of the main theorem . . . . . . . . . . . . . . . . . 10

2.5 Proof of Lemma $2.3 .5 \ldots \ldots \ldots \ldots$

3 Hadwiger's Conjecture for Claw-free Graphs 33

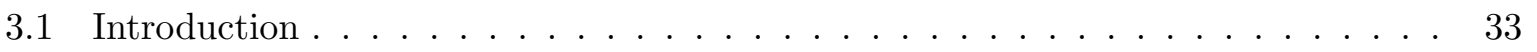

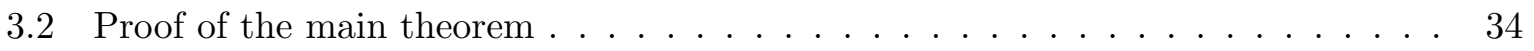


4.1 Introduction . . . . . . . . . . . . . . . . . . . 47

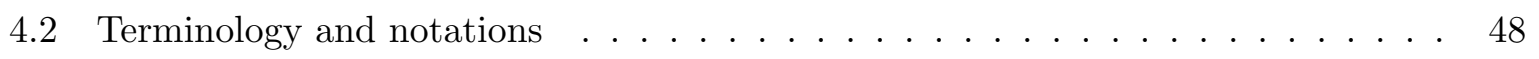

4.3 Lemmas . . . . . . . . . . . . . . . . . . . . . . 49

4.4 Proof of the main theorem . . . . . . . . . . . . . . 50 


\section{Chapter 1}

\section{Introduction}

In this dissertation, we study problems related to Hadwiger's Conjecture and Thomassen's Conjecture.

In 1943, Hadwiger made the following conjecture, which is a generalization of the Four Color Problem.

Conjecture 1.0.1 (Hadwiger [10]) For all $k \geq 1$, every $k$-chromatic graph has the complete graph $K_{k}$ on $k$ vertices as a minor.

A graph $H$ is a minor of a graph $G$ if $H$ can be obtained from $G$ by deleting edges and vertices and contracting edges.

In Chapter 2, we give the following result.

Theorem 2.1.12 Let $G$ be a $(k+2)$-connected, non- $(k-3)$-apex graph where $k \geq 2$. If $G$ contains three $k$-cliques, say $L_{1}, L_{2}, L_{3}$, such that $\left|L_{i} \cap L_{j}\right| \leq k-2(1 \leq i<j \leq 3)$, then $G$ contains a $K_{k+2}$ as a minor.

In Chapter 3, we give the following result.

Theorem 3.1.6 Let $G$ be a 6-connected claw-free graph. If $\delta(G) \geq 7$ and $G$ contains three disjoint 5 -cliques, say $L_{1}, L_{2}, L_{3}$, then $G$ contains a $K_{7}$ as a minor. 
In 1985, Thomassen made the following conjecture.

Conjecture 1.0.2 (Thomassen [1], p. 466) Every longest circuit of a 3-connected graph must have a chord.

An edge $e$ is called a chord of a circuit if $e$ is not an edge of the circuit and both end vertices of $e$ are in the circuit.

Thomassen's conjecture has been verified for many families of graphs. In Chapter 4, we verify the conjecture for locally planar graphs, and we prove the following result.

Theorem 4.1.2 There is a function $h: N \longrightarrow N$, such that, for every 4-connected graph $G$ with minimum degree at least five embedded in a surface with Euler genus $g$ and face-width at least $h(g)$, every longest circuit of the graph $G$ has a chord. 


\section{Chapter 2}

\section{Cliques, Minors and Apex Graphs}

\subsection{Introduction}

Hadwiger's Conjecture from 1943 suggests a far reaching generalization of the Four Color Problem, and it is one of the most famous problems in the theory of graph minors. Hadwiger's Conjecture states the following.

Conjecture 2.1.1 (Hadwiger [10]) For all $k \geq 1$, every $k$-chromatic graph has the complete graph $K_{k}$ on $k$ vertices as a minor.

For $k=1,2,3$, this conjecture is easy to prove, and for $k=4$, Hadwiger [10] and Dirac [9] proved it. For $k=5$, however, it seems extremely difficult. In 1937, Wagner [35] proved that the case $k=5$ is equivalent to the Four Color Theorem [2, 3, 24]. In 1993, Robertson, Seymour and Thomas [27] proved that a minimal counterexample to the case $k=6$ is a graph $G$ which has a vertex $v$ such that $G-v$ is planar. Hence, assuming the Four Color Theorem, the case $k=6$ of Hadwiger's Conjecture holds. This result is the deepest in this research area. So far, the cases $k \geq 7$ are open.

Motivated by Hadwiger's Conjecture, the following question has drawn the attention of many researchers. 
Question 2.1.2 What do $K_{k}$-minor-free graphs look like?

One approach to this question is to consider the maximal size of graphs not having $K_{k}$ as a minor. Wagner [36] showed that a sufficiently large chromatic number (which depends only on $k$ ) guarantees a $K_{k}$ as a minor, and Mader [20] showed that a sufficiently large average degree will do. Kostochka [17] and Thomason [30] independently proved that $k \sqrt{\log k}$ is the correct order for the average degree because random graphs having no $K_{k}$-minor may have average degree of order $k \sqrt{\log k}$. (Recently, Thomason [31] gave a more exact "extremal" function.)

Another approach is due to Robertson and Seymour [25]. They considered how to construct graphs with no $K_{k}$-minor. If $G$ contains a set $X$ with at most $k-5$ vertices such that $G-X$ is planar, $G$ does not contain $K_{k}$ as a minor since planar graphs cannot contain $K_{5}$ as a minor. Similarly, if $G$ contains a set $X$ with at most $k-7$ vertices such that $G-X$ can be drawn in the projective plane, then clearly $G$ does not contain $K_{k}$ as a minor. (Since graphs drawn on the projective plane cannot contain $K_{7}$ as a minor.) Or if $G$ contains a set $X$ with at most $k-8$ vertices such that $G-X$ can be drawn in the torus, then clearly $G$ does not contain $K_{k}$ as a minor. (Again, graphs drawn on the torus cannot contain $K_{8}$ as a minor.) These observations together with the concept "clique-sum" led Robertson and Seymour to one of their celebrated results of excluding complete graph minors, and this is the most important step in their proof of "Wagner's Conjecture" [26].

Our motivation is the following question.

Question 2.1.3 What do $K_{k}$-minor-free $k$-connected graphs look like?

It does not seem that random graphs give an answer to this question because, as Thomason [31] pointed out, extremal graphs are more or less exactly vertex disjoint unions of suitable dense random graphs. It does not seem that Robertson and Seymour's excluded minor theorem gives an answer either, because their characterization does not seem to guarantee high connectivity. In view of these observations, we still do not know what $K_{k}$-minor-free $k$-connected graphs look like.

The following question is motivated by Hadwiger's Conjecture.

Question 2.1.4 Is it true that a minimal counterexample to Hadwiger's Conjecture for $k \geq 6$ has a set $X$ of $k-5$ vertices such that $G-X$ is planar?

This is true for $k=6$ as Robertson, Seymour and Thomas [27] showed. To consider a minimal counterexample to Hadwiger's Conjecture, one can prove the following conjecture. 
Conjecture 2.1.5 A minimal counterexample to Hadwiger's Conjecture is $k$-connected.

This is true for $k \leq 7$ as Mader proved in [21]. Note that Toft [34] proved that a minimal counterexample to Hadwiger's Conjecture is $k$-edge-connected. This is strong evidence for Conjecture 2.1.5.

Question 2.1.4 and Conjecture 2.1.5 lead us to the following question.

Question 2.1.6 Is it true that a $K_{k}$-minor-free $k$-connected graph for $k \geq 6$ has a set $X$ of $k-5$ vertices such that $G-X$ is planar?

The case $k=6$ is a well-known conjecture due to Jorgensen [13], and is still open. If true, this would imply Hadwiger's Conjecture for the $k=6$ case by Mader's result [20]. The case $k=7$ was conjectured in [14] as well.

Even though the case $k=6$ of the Question 2.1.6 is still open, Robertson, Seymour and Thomas [27] gave a result for searching for a $K_{6}$-minor.

Theorem 2.1.7 (Robertson, Seymour and Thomas [27]) Let $G$ be a simple 6-connected nonapex graph. If $G$ contains three 4 -cliques, say, $L_{1}, L_{2}, L_{3}$, such that $\left|L_{i} \cap L_{j}\right| \leq 2(1 \leq i<j \leq 3)$, then $G$ contains a $K_{6}$ as a minor.

Recently, Kawarabayashi and Toft [14] proved the following theorem.

Theorem 2.1.8 (Kawarabayashi and Toft [14]) Any 7 -chromatic graph has $K_{7}$ or $K_{4,4}$ as a minor.

This settles the case $(6,1)$ of the following conjecture known as the $(k-1,1)$-Minor Conjecture, which is a relaxed version of Hadwiger's Conjecture.

Conjecture 2.1.9 (Chartrand, Geller, Hedetniemi [7]; Woodall [37]) For all $k \geq 1$, every $k$ chromatic graph has either a $K_{k}$-minor or a $K_{\left\lfloor\frac{k+1}{2}\right\rfloor,\left\lceil\frac{k+1}{2}\right\rceil}$-minor. 
In [14], the following result is the key lemma, which gives a result for searching for a $K_{7^{-}}$ minor.

Theorem 2.1.10 (Kawarabayashi and Toft [14]) Let $G$ be a 7-connected graph. Suppose $G$ contains three 5-cliques, say, $L_{1}, L_{2}, L_{3}$, such that $\left|L_{1} \cup L_{2} \cup L_{3}\right| \geq 12$, then $G$ contains a $K_{7}$-minor.

Recently, Kawarabayashi, R. Luo, J. Niu and C-Q Zhang [15] proved the following theorem.

Theorem 2.1.11 (Kawarabayashi, R. Luo, J. Niu and C-Q Zhang [15]) Let $G$ be a $(k+2)$ connected graph where $k \geq 5$. If $G$ contains three $k$-cliques, say $L_{1}, L_{2}, L_{3}$, such that $\mid L_{1} \cup L_{2} \cup$ $L_{3} \mid \geq 3 k-3$, then $G$ contains a $K_{k+2}$ as a minor.

Our work is motivated by Theorem 2.1.7, and the main result of this Chapter is the following theorem which generalizes Theorem 2.1.7, Theorem 2.1.10 and Theorem 2.1.11.

Theorem 2.1.12 Let $G$ be a $(k+2)$-connected, non- $(k-3)$-apex graph where $k \geq 2$. If $G$ contains three $k$-cliques, say $L_{1}, L_{2}, L_{3}$, such that $\left|L_{i} \cap L_{j}\right| \leq k-2(1 \leq i<j \leq 3)$, then $G$ contains a $K_{k+2}$ as a minor.

We hoped our result could be used to prove some results on 7 - and 8-chromatic graphs. In fact, in [16], Kawarabayashi proved that any 7 -chromatic graph has $K_{7}$ or $K_{3,5}$ as a minor using Theorem 2.1.11. Maybe one can use this result to prove 8-chromatic case of Conjecture 2.1.9.

A $k$-connected graph may contain many $(k-2)$-cliques, but not necessarily a $K_{k}$-minor. For example, the graph $K_{k-5}+G_{1}$, where $G_{1}$ is a 5-connected planar graph, is $K_{k}$-minorfree and contains many copies of $(k-2)$-cliques. Theorem 2.1.12, implies that a $k$-connected $K_{k}$-minor-free graph can't contain three "nearly" disjoint $(k-2)$-cliques.

The following was conjectured by Seymour and Thomas (private communication with R. Thomas.)

Conjecture 2.1.13 For every $p \geq 1$, there exists a constant $N=N(p)$ such that every $(p-2)$ connected graph on $n \geq N$ vertices and at least $(p-2) n-\frac{(p-1)(p-2)}{2}+1$ edges has a $K_{p}$-minor. 
Note that the connectivity condition and the condition of the order of graphs are necessary because random graphs having no $K_{k}$-minor may have average degree $k \sqrt{\log k}$, but all these graphs are small. So if a graph is large enough and highly connected, we do not know any infinite family of counterexamples. This conjecture is true for $p \leq 9$. For $p \leq 7$, these conjecture were proved by Mader [21]. Jorgensen [13] proved the case of $p=8$. Very recently, Song and Thomas [29] proved the case $p=9$. Note that all of these results do not require the connectivity condition in this conjecture.

We hope that our result could give a weaker result since, as far as we know, the only known extremal graphs are $K_{k-5}+G_{1}$, where $G_{1}$ is a 5-connected planar graph. So this graph could contain $(k-2)$-clique. On the other hand, our result implies that it cannot contain three nearly "disjoint" $K_{k-2}$. Hence one can prove a weaker bound on the number of edges.

\subsection{Terminology and notations}

All graphs considered in this paper are finite, undirected, and without loops or multiple edges. The complete graph (or, clique, as a subgraph) on $n$ vertices is denoted by $K_{n}$ and the complete bipartite graph such that one partite set has $n$ vertices and the other partite set has $m$ vertices is denoted by $K_{n, m}$.

A graph $H$ is a minor of a graph $G$ if $H$ can be obtained from $G$ by deleting edges and vertices and contracting edges.

For a vertex $x$ of a subgraph $H_{1}$ of $G$, the neighborhood of $x$ in $H_{1}$ is denoted by $N_{H_{1}}(x)$. For a vertex $v \in V(G)$ and a vertex subset (or a subgraph) $Y$ of $G, d_{Y}(x)=|\{v \in Y: x v \in E(G)\}|$. A graph $G$ is $k$-chromatic if $G$ is vertex- $k$-colorable, but not vertex- $(k-1)$-colorable. Let $V_{1}$ and $V_{2}$ be subsets of $V(G)$. The symmetric difference of $V_{1}$ and $V_{2}$, denoted by $V_{1} \Delta V_{2}$, is the set $\left(V_{1} \cup V_{2}\right)-\left(V_{1} \cap V_{2}\right)$.

We say a graph $G$ is $k$-apex if $G-X$ is planar for some subset $X \subseteq V(G)$ with $|X|=k$. By the definition, a 0 -apex graph is planar. A 1-apex graph is often referred as an apex graph if there is no confusion.

A subset $X \subseteq V(G)$ is a fragment of $G$ if $X \neq \emptyset$ and $X$ induces a connected subgraph of $G$. Subsets $X, Y \subseteq V(G)$ are adjacent in $G$ if $X \cap Y=\emptyset$ and some $x \in X$ is adjacent in $G$ to some $y \in Y$.

A cluster in $G$ is a set of mutually adjacent fragments $G$, and it is a p-cluster if it has cardinality $p$. Thus, $G$ has a $K_{p}$-minor if and only if it has a $p$-cluster. Given a subset $Y \subseteq V(G)$, a $p$-cluster $\wp$ is said to traverse $Y$ if $\wp=\left\{X_{1}, X_{2}, \cdots, X_{p}\right\}$ and $X_{i} \cap Y \neq \emptyset(1 \leq i \leq q)$. 
Let $v_{1}, v_{2}, v_{3}$ be mutually adjacent vertices of a graph $G$. We say $G$ is triangular with respect to $v_{1}, v_{2}, v_{3}$ if $G$ is simple and either

(i) for some $i(1 \leq i \leq 3), G-v_{i}$ has maximum degree at most 2 , and either $G-v_{i}$ is a cycle or it has no cycle, or

(ii) all vertices of $G$ have degree at most 3 , there is at most one vertex $v$ of degree 3 with $v \neq v_{1}, v_{2}, v_{3}$, and $G-v_{1}-v_{2}-v_{3}$ has no cycle, or

(iii) all vertices of $G$ have degree at most 3, there is a triangle $C$ in $G-v_{1}-v_{2}-v_{3}$, every vertex of degree 3 is in $\left\{v_{1}, v_{2}, v_{3}\right\} \cup V(C)$, and every cycle except for the two triangles $\left\{v_{1}, v_{2}, v_{3}\right\}$ and $C$ contains both a vertex in $\left\{v_{1}, v_{2}, v_{3}\right\}$ and $V(C)$.

\subsection{Lemmas}

\subsubsection{Good paths}

One of the key lemmas in our proof is Mader's " $H$-Wege" Theorem, which was proved in [22].

Lemma 2.3.1 (Mader [22]) Let $G$ be a graph, let $S \subseteq V(G)$ be an independent set, and $k \geq 0$ be an integer. Then exactly one of the following two statements holds.

(1) There are $k$ paths of $G$, each with two distinct ends both in $S$, such that each $v \in V(G)-S$ is in at most one of the paths.

(2) There exists a vertex set $W \subseteq V(G)-S$ and a partition $Y_{1}, \cdots, Y_{n}$ of $V(G)-(S \cup W)$, and a subset $X_{i} \subseteq Y_{i}, 1 \leq i \leq n$, such that

(a) $|W|+\sum_{1 \leq i \leq n}\left\lfloor\frac{1}{2}\left|X_{i}\right|\right\rfloor<k$,

(b) no vertex in $Y_{i}-X_{i}$ has a neighbor in $V(G)-\left(W \cap Y_{i}\right)$ and,

(c) every path of $G-W$ with distinct ends both in $S$ has an edge with both ends in $Y_{i}$ for some $i$. 
Let $Z_{1}, Z_{2}, \cdots, Z_{h}$ be subsets of $V(G)$. A path $P$ of $G$ with ends $u, v$ is said to be good if there exist distinct $i, j$ with $1 \leq i, j \leq h$ such that $u \in Z_{i}$ and $v \in Z_{j}$.

As Robertson, Seymour and Thomas pointed out in [27], we can deduce the following lemma from Lemma 2.3.1.

Lemma 2.3.2 (Robertson, Seymour and Thomas [27]) Let $G$ be a graph, let $Z_{1}, Z_{2}, \cdots, Z_{h}$ be subsets of $V(G)$, and let $k \geq 1$ be an integer. Then exactly one of the following two statements holds.

(1) There are $k$ mutually disjoint good paths of $G$.

(2) There exists a vertex set $W \subseteq V(G)$ and a partition $Y_{1}, \cdots, Y_{n}$ of $V(G)-W$, and a subset $X_{i} \subseteq Y_{i}$, for $1 \leq i \leq n$ such that

(a) $|W|+\sum_{1 \leq i \leq n}\left\lfloor\frac{1}{2}\left|X_{i}\right|\right\rfloor<k$,

(b) for any $i$ with $1 \leq i \leq n$, no vertex in $Y_{i}-X_{i}$ has a neighbor in $V(G)-\left(W \cup Y_{i}\right)$ and $Y_{i} \cap\left(\cup_{j=1}^{h} Z_{j}\right) \subseteq X_{i}$, and

(c) every good path $P$ in $G-W$ has an edge with both ends in $Y_{i}$ for some $i$.

\subsubsection{Cluster}

Lemma 2.3.3 (Robertson, Seymour and Thomas [27], page 291) Let $v_{1}, v_{2}, v_{3}$ be mutually adjacent vertices of a 4-connected simple non-planar graph $G$. Let $\Im \subseteq V(G)$ with $v_{1}, v_{2}, v_{3} \in \Im$ such that $\Im$ is not triangular. Then there is a 5-cluster $\wp=\left\{\left\{v_{1}\right\},\left\{v_{2}\right\},\left\{v_{3}\right\}, X_{1}, X_{2}\right\}$ in $G$ such that $\wp$ traverses $\Im$.

The following lemma is an immediate corollary of a result by Robertson, Seymour and Thomas in [27] (page 288).

Lemma 2.3.4 Let $G$ be a 4-connected graph and $\Im \subseteq V(G)$ with $|\Im|=4$. Then either 
(i) there is a 4-cluster in $G$ traversing $\Im$, or

(ii) $G$ can be drawn in a plane so that every vertex in $\Im$ is incident with the infinite region.

\subsubsection{The 6-cluster lemma}

The following lemma deals with an extreme case of our main theorem (Theorem 2.1.12). Since the proof of the lemma is relatively long and complicated, we present it here as an independent lemma and its proof in Section 2.5, Readers may postpone the reading of the proof of Lemma 2.3.5 until after the proof of the main theorem.

Lemma 2.3.5 Let $x_{i}, y_{i}, z_{i}(1 \leq i \leq 3)$ be distinct vertices of a 6-connected simple graph $G$, such that $\left\{x_{1}, y_{1}, z_{2}, z_{3}\right\},\left\{x_{2}, y_{2}, z_{3}, z_{1}\right\},\left\{x_{3}, y_{3}, z_{1}, z_{2}\right\}$ are 4-cliques. Suppose, that there is a partition $Y_{1}, Y_{2}$ of $V(G)-\left\{z_{1}, z_{2}, z_{3}\right\}$ with $x_{1}, x_{2}, x_{3} \in Y_{1}$, and $y_{1}, y_{2}, y_{3} \in Y_{2}$, such that $x_{i} y_{i}$ $(1 \leq i \leq 3)$ are the only edges of $G$ with one end in $Y_{1}$ and the other in $Y_{2}$. Then $G$ has a 6 -cluster traversing $\left\{x_{1}, y_{1}, z_{1}, x_{2}, y_{2}, z_{2}, x_{3}, y_{3}, z_{3}\right\}$.

Note that, Robertson, Seymour and Thomas gave a result in page 293 of [27] similar to Lemma 2.3.5. However, in order to obtain a sharper and more general result in our main theorems (Theorem 2.1.12 and Theorem 2.4.1), we need a stronger result in Lemma 2.3 .5 (for 6-cluster instead of 6-minor), which is approached differently from that in [27].

\subsection{Proof of the main theorem}

The main theorem (Theorem 2.1.12) is to be proved in this section. Here we prove a theorem that is slightly stronger than the main theorem (Theorem 2.1.12).

Theorem 2.4.1 Let $G$ be a $(k+2)$-connected, non-( $k-3)$-apex graph where $k \geq 2$. If $G$ contains three $k$-cliques, say $L_{1}, L_{2}, L_{3}$, such that $\left|L_{i} \cap L_{j}\right| \leq k-2(1 \leq i<j \leq 3)$, then one of the following holds, 
(1) G contains a $(k+2)$-cluster traversing $L_{1} \cup L_{2} \cup L_{3}$, or

(2) (an exceptional case) $|T|=k-2$ where $T=L_{1} \cap L_{2} \cap L_{3}$, and $G-T$ is a planar graph with all edges $L_{i}-T(i=1,2,3)$ around the exterior face. In this case, $G$ contains a $(k+2)$-cluster $\left\{\left\{v_{1}\right\}, \cdots,\left\{v_{k}\right\}, B, I\right\}$ where $L_{1}=\left\{v_{1}, \cdots, v_{k}\right\}, B$ is the set of all vertices of $G-T$ around the exterior face except for those in $L_{1}$, and $I$ is the set of all interior vertices of $G-T$.

Note: readers might be confused by a non- $(k-3)$-apex graph if $k=2$. Recall that a graph $H$ is a non-t-apex if $G-R$ is planar for some vertex subset $R$, then $R$ must be of order at least $t+1$. Hence, a non- $(k-3)$-apex graph for $k=2$ can be any graph, planar or non-planar.

Proof: Let $G$ be a counterexample to the theorem with $k$ as small as possible.

\subsubsection{We claim that $k \geq 3$.}

For otherwise, we may assume $k=2, G$ is 4-connected graph, and $G$ contains three disjoint 2-cliques, say $L_{1}, L_{2}, L_{3}$.

Since $L_{1}$ and $L_{2}$ are disjoint 2-cliques, $\left|L_{1} \cup L_{2}\right|=4$. Note that $G$ is 4-connected, by Lemma 2.3.4. There are two cases:

(1) There is a 4-cluster in $G$ traversing $L_{1} \cup L_{2}$. In this case, by the definition of cluster, this 4-cluster in $G$ also traverses $L_{1} \cup L_{2} \cup L_{3}$, hence we are done.

(2) $G$ can be drawn in a plane so that every vertex in $L_{1} \cup L_{2}$ is incident with the infinite region. In this case, since $G$ is 4 -connected, the edges of $L_{1}$ and $L_{2}$ must be around the exterior face. If one vertex $v_{1}$ of $L_{3}$ is not incident with the infinite region, then there are four internal vertex-disjoint paths from $v_{1}$ to $L_{1} \cup L_{2}$, hence we get a 4-cluster traversing $L_{1} \cup L_{2} \cup L_{3}$. Therefore two vertices of $L_{3}$ must be incident with the infinite region. Note that $G$ is 4-connected, $G$ has a 4-cluster $\left\{\left\{v_{1}\right\},\left\{v_{2}\right\}, B, I\right\}$ where $L_{1}=\left\{v_{1}, v_{2}\right\}, B$ is the set of all vertices of $G$ around the exterior face except for those in $L_{1}$, and $I$ is the set of all interior vertices of $G$ (it is easy to see that $I$ and $B$ both are connected).

2.4.2 We claim that $\left|L_{1} \cap L_{2} \cap L_{3}\right|=0$. 
For otherwise, we assume $\left|L_{1} \cap L_{2} \cap L_{3}\right| \neq 0$. Let $x \in L_{1} \cap L_{2} \cap L_{3}$ and $G^{\prime}=G-\{x\}$, then $G^{\prime}$ is a $(k+1)$-connected non $(k-4)$-apex graph. By minimality of $k$, there are two cases:

Case (1): There is a $(k+1)$-cluster $\wp_{1}$ of $G-\{x\}$ traversing $L_{1} \cup L_{2} \cup L_{3}-\{x\}$. Let $\wp=\wp_{1} \cup\{\{x\}\}$, then $\wp$ is a $(k+2)$-cluster traversing $L_{1} \cup L_{2} \cup L_{3}$, a contradiction.

Case (2): $\left|T^{\prime}\right|=k-3$ where $T^{\prime}=\left(L_{1} \cap L_{2} \cap L_{3}\right)-\{x\}$, and $G^{\prime}-T^{\prime}$ is a planar graph with all edges of $\left(L_{i}-\{x\}\right)-T^{\prime}$ around the exterior face. $G^{\prime}$ contains a $(k+1)$-cluster $\wp^{\prime}=$ $\left\{\left\{v_{1}\right\}, \cdots,\left\{v_{k-1}\right\}, B, I\right\}$ where $\left\{x, v_{1}, \cdots, v_{k-1}\right\}=L_{1}$ and $B$ is the set of all vertices of $G^{\prime}-T^{\prime}$ around the exterior face except for those in $L_{1}$, and $I$ is the set of all interior vertices of $G^{\prime}-T^{\prime}$.

In order to show that $\wp=\left\{\{x\},\left\{v_{1}\right\}, \cdots,\left\{v_{k-1}\right\}, B, I\right\}$ is a $(k+2)$-cluster of $G$, it is sufficient to prove that $x$ is adjacent to every other fragment. (i) it is obvious that $v_{i} \in N(x)$ since $x \in L_{1}$; (ii) it is similar that $N(x) \cap B \neq \emptyset$ since $x \in L_{2}$ and $L_{2} \cap B \neq \emptyset$; (iii) $N(x) \cap I \neq \emptyset$ for otherwise, the vertex $x$ can be embedded into the exterior face of $G^{\prime}-T^{\prime}$ and therefore, $G-T^{\prime}$ is planar. This contradicts that $G$ is non- $(k-3)$-apex.

2.4.3 We claim that $k \geq 4$.

For otherwise, by (2.4.1), we may assume $k=3$. That is, $G$ is a 5-connected non-planar graph, and $G$ contains three 3 -cliques, say $L_{1}, L_{2}, L_{3}$, such that $\left|L_{i} \cap L_{j}\right| \leq 1(1 \leq i<j \leq 3)$. By (2.4.2), we have $\left|L_{1} \cap L_{2} \cap L_{3}\right|=0$.

Let $Z=L_{1} \cup L_{2} \cup L_{3}$ and $v_{1}, v_{2}, v_{3} \in L_{1}$. Then $Z$ is not triangular with respect to $v_{1}, v_{2}$, $v_{3}$. By Lemma 2.3.3, there is a 5 -cluster $\wp$ in $G$ such that $\wp$ traverses $Z$.I

2.4.4 We claim that $G$ does not contain a $(k+2)$-cluster traversing $L_{1} \cup L_{2} \cup L_{3}$.

By (2.4.2), we have $\left|L_{1} \cap L_{2} \cap L_{3}\right|=0$. And by (2.4.3), $k \geq 4$. Hence $G$ is at least 6 -connected, $|E(G)| \geq 3|V(G)|$. Therefore $G$ is not a planar graph, that is, $G$ is not the exceptional case. Hence the result follows.

2.4.5 We claim that $\left|L_{i} \cap L_{j}\right| \leq 1$ for $1 \leq i<j \leq 3$.

For otherwise, we may assume $\left|L_{1} \cap L_{2}\right| \geq 2$. Let $B \subseteq L_{1} \cap L_{2}$ with $|B|=2$. By (2.4.2), $B \cap L_{3}=\emptyset$ since $L_{1} \cap L_{2} \cap L_{3}=\emptyset$. Since $G-B$ is $k$-connected, there exist $k$ disjoint paths 
from $L_{3}$ to $L_{1} \cup L_{2}-B$. Let $x, y \in B$ and $P_{1}, P_{2}, \cdots, P_{k}$ be a set of disjoint paths from $L_{3}$ to $L_{1} \cup L_{2}-B$. Then $\wp=\left\{P_{1}, P_{2}, \cdots, P_{k}, x, y\right\}$ is a $(k+2)$-cluster that traverses $L_{1} \cup L_{2} \cup L_{3}$, a contradiction.

2.4.6 A path $P$ of $G$ with ends $u, v$ is said to be good if there exist distinct $i, j$ with $1 \leq i, j \leq 3$ such that $u \in L_{i}$ and $v \in L_{j}$.

2.4.7 We claim that there don't exist $(k+2)$ mutually disjoint good paths in $G$.

Let $P_{1}, P_{2}, \cdots, P_{k+2}$ be a set of disjoint good paths of $G$. Then $\wp=\left\{P_{1}, P_{2}, \cdots, P_{k+2}\right\}$ is a $(k+2)$-cluster that traverse $L_{1} \cup L_{2} \cup L_{3}$.

By Lemma 2.3.2 and (2.4.7), we have the following structure of $G$ :

2.4.8 There exists a vertex set $W \subseteq V(G)$ and a partition $Y_{1}, \cdots, Y_{n}$ of $V(G)-W$, and a subset $X_{i} \subseteq Y_{i}$, for $1 \leq i \leq n$ such that

(a) $|W|+\sum_{1 \leq i \leq n}\left\lfloor\frac{1}{2}\left|X_{i}\right|\right\rfloor \leq k+1$,

(b) for any $i$ with $1 \leq i \leq n$, no vertex in $Y_{i}-X_{i}$ has a neighbor in $V(G)-\left(W \cup Y_{i}\right)$ and $Y_{i} \cap\left(\cup_{j=1}^{3} L_{j}\right) \subseteq X_{i}$, and

(c) every good path $P$ in $G-W$ has an edge with both ends in $Y_{i}$ for some $i$.

Let $M=\left(L_{1} \cap L_{2}\right) \cup\left(L_{2} \cap L_{3}\right) \cup\left(L_{3} \cap L_{1}\right)$, and choose $W$ and $Y_{1}, X_{1}, \cdots, Y_{n}, X_{n}$ such that $|W|$ is as large as possible. Without loss of generality, we can assume that $Y_{i} \neq \emptyset$ for any $i \in\{1,2, \cdots, n\}$. By the definition of $W, M$ and $(2.4 .8)(\mathrm{c})$, we have the following immediate observations:

\section{4 .9}

(a) $\quad M \subseteq W$

by $(2.4 .8)(\mathrm{c})$. 
(b) $\quad\left|L_{1} \cup L_{2} \cup L_{3}\right|=\left|L_{1}\right|+\left|L_{2}\right|+\left|L_{3}\right|-|M|$

by definition of $M$ and (2.4.2).

(c) $\quad|M| \leq 3$

by (2.4.2) and (2.4.5).

(d) $\quad\left|L_{i} \cup L_{j}\right|>k+2$ for $1 \leq i<j \leq 3$.

$(2.4 .9)(\mathrm{d})$ is proved as follows: by $(2.4 .5)$ and $k \geq 4$ (by (2.4.3))

$\left|L_{i} \cup L_{j}\right|=\left|L_{i}\right|+\left|L_{j}\right|-\left|L_{i} \cap L_{j}\right|=2 k-1>k+2$.

The following claim (e) follows from the assumption (2.4.8)(b).

(e)

$$
W \cup X_{1} \cup \cdots \cup X_{n} \supseteq L_{1} \cup L_{2} \cup L_{3} \text {, and }|W|+\sum_{i=1}^{n}\left|X_{i}\right| \geq\left|L_{1} \cup L_{2} \cup L_{3}\right| \text {. }
$$

2.4.10 We claim that $X_{i} \neq \emptyset$ for all $i$.

Suppose that $X_{i}=\emptyset$ for some $i$. Since $|W| \leq k+1$ (by (2.4.8)(a)) and $\left|L_{1} \cup L_{2} \cup L_{3}\right| \geq$ $\left|L_{1} \cup L_{2}\right| \geq k+2$ (by (2.4.9)(d)), there is an integer $j(j \neq i)$ such that $X_{j} \neq \emptyset$ (by (2.4.9)(e)). Hence $n \geq 2$. Since $Y_{i}$ is not empty, $W$ is a cutset that separates $Y_{i}$ and non-empty $X_{j}$ and is of cardinality at most $k+1$. This contradicts that $G$ is $(k+2)$-connected.

2.4.11 We claim that $\left|X_{i}\right|$ is odd for all $i$.

Suppose that $\left|X_{1}\right|$ is even, then by (2.4.10), $\left|X_{1}\right| \geq 2$. Let $v \in X_{1}, W^{*}=W \cup\{v\}$, $Y_{1}^{*}=Y_{1}-v, X_{1}^{*}=X_{1}-v$ and $X_{i}^{*}=X_{i}, Y_{i}^{*}=Y_{i}$ for $2 \leq i \leq n$. The partition $\left\{W^{*}, X_{1}^{*}, \cdots\right.$, $\left.X_{n}^{*}, Y_{1}^{*}, \cdots, Y_{n}^{*}\right\}$ of $V(G)$ satisfies $(2.4 .8)(\mathrm{a})$ - (c), contradicting the choice that $|W|$ is as large as possible.

\subsubsection{We claim that}

(a) $\quad n \geq k-2$.

(b) if $n=k-2$ then

$$
|W|=|M| \text { and } W \cup X_{1} \cup X_{2} \cup \cdots \cup X_{n}=L_{1} \cup L_{2} \cup L_{3} \text {. }
$$


Since $\left|L_{1} \cup L_{2} \cup L_{3}\right| \geq\left|L_{1} \cup L_{2}\right| \geq k+2$ (by (2.4.9)(d)) and $|W| \leq k+1$ (by (2.4.8)(a)), we have $n \geq 1$. By (2.4.8)(a), (2.4.9)(a) and (2.4.9)(b), we have

$$
\begin{aligned}
2(k+1) & \geq 2\left(|W|+\sum_{1 \leq i \leq n}\left\lfloor\frac{1}{2}\left|X_{i}\right|\right\rfloor\right)=2|W|+\sum_{1 \leq i \leq n}\left|X_{i}\right|-n \\
& \geq|W|+\left|L_{1} \cup L_{2} \cup L_{3}\right|-n \geq|M|+\left|L_{1} \cup L_{2} \cup L_{3}\right|-n \\
& =\left|L_{1}\right|+\left|L_{2}\right|+\left|L_{3}\right|-n=3 k-n .
\end{aligned}
$$

Thus,

$$
n \geq k-2
$$

and if $n=k-2$, then all equalities hold and therefore,

$$
|W|=|M| \text { and }|W|+\sum_{1 \leq i \leq n}\left|X_{i}\right|=\left|L_{1} \cup L_{2} \cup L_{3}\right| \text {. }
$$

\subsubsection{Definition of $A_{i}($ for $i=1,2,3)$}

Let $G^{\prime \prime}$ be the subgraph obtained from $G-W$ by deleting all edges contained in any $Y_{j}$. Let $A_{i}$ be the union of the vertex subsets of all components of $G^{\prime \prime}$ containing some vertex of $L_{i}$ for each $i \in\{1,2,3\}$.

\subsubsection{Properties of $\left\{A_{1}, A_{2}, A_{3}\right\}$}

Properties of $\left\{A_{1}, A_{2}, A_{3}\right\}$ are to be studied in this subsection. The first property is immediate by (2.4.8) and the definition of $A_{i}$.

(a) $L_{i}-W \subseteq A_{i} \subseteq V(G)-W$ for $i=1,2,3$.

Note that each $Y_{j}-X_{j}$ is an independent set of $G^{\prime \prime}$, and by $(2.4 .8)(\mathrm{b})$, we have the following properties.

(b) $A_{i} \subseteq X_{1} \cup \cdots \cup X_{n}$ for $i=1,2,3$.

(c) $A_{1}, A_{2}, A_{3}$ are disjoint by the definition of $A_{i}$ and $(2.4 .8)(\mathrm{c})$. 
(d) Every path of $G-W$ from $A_{i}$ to $A_{i^{*}}$ (for $1 \leq i<i^{*} \leq 3$ ) has at least two vertices in $X_{j}$ for some $j$.

Proof of (d). Suppose there exists a path $P$ from $v \in A_{1}$ to $u \in A_{2}$ in $G-W$. By the definition of $A_{1}, A_{2}$, we can take two disjoint paths $Q$ and $R$ such that $Q$ is a path from some vertex $x \in L_{1}$ to $v$ in $G\left[A_{1}\right]$ and $R$ is a path from some vertex $y \in L_{2}$ to $u$ in $G\left[A_{2}\right]$. Both $Q$ and $R$ have no edges with both ends in $Y_{j}$ for any $j$ by definition of $A_{i}$. Then we have a path $S$ from $x$ to $y$ by using $Q, P, R$. Since $S$ is a good path by (2.4.8)(c), $S$ has an edge $e=x_{1} y_{1} \in Y_{j}$ for some $j$. Note that $e \notin E(Q)$ and $e \notin E(R)$. This implies $e \in E(P)$ and $x_{1}, y_{1} \in V(P)$. Note that, by (2.4.14)(b), both $v$ and $u$ belong to $X_{1} \cup \cdots \cup X_{n}$. By (2.4.8)(b), the part of $P$ from $v$ to $x_{1}$ must contain a vertex from $X_{j}$, and likewise the part of $P$ from $y_{1}$ to $u$.

(e) $\left|A_{i}\right| \leq k+1-|W|$ for $1 \leq i \leq 3$.

Proof of (e). Suppose $\left|A_{1}\right| \geq k+2-|W|$. It is obvious that $|W| \leq k+1$ (by (2.4.8)(a)). Hence, $A_{1} \neq \emptyset$. We also have that $L_{2} \cup L_{3}-W \neq \emptyset$ since $\left|L_{2} \cup L_{3}\right| \geq k+2$ (by (2.4.9)(d)) and $|W| \leq k+1$ (by $(2.4 .8)(\mathrm{a}))$.

Since $\left|L_{2} \cup L_{3}\right| \geq k+2$ (by $\left.(2.4 .9)(\mathrm{d})\right)$, we have that $\left|L_{2} \cup L_{3}-W\right| \geq k+2-|W|$. Note that $G-W$ is $(k+2-|W|)$-connected, there are $(k+2-|W|)$ disjoint paths from $A_{1}$ to $L_{2} \cup L_{3}-W$ each of which is of order at least $k+2-|W|$. By (2.4.14)(d), every path $P_{j}$ contains at least two vertices of $X_{i}$ for some $i$. Hence, $\sum_{1 \leq i \leq n}\left\lfloor\frac{1}{2}\left|X_{i}\right|\right\rfloor \geq k+2-|W|$. This is a contradiction to $(2.4 .8)(a)$. The other cases follow by similar argument.

2.4.15 We claim that $|W| \leq 3$. If equality holds then $M=W$ and $\left|A_{i}\right|=k+1-|W|$.

This claim is to be proved in two steps in this subsection. First we show that

$$
\sum_{i=1}^{3}\left|L_{i} \cap W\right| \leq|W|+3
$$

Note that $\sum_{i=1}^{3}\left|L_{i} \cap W\right| \leq|W|+|M|$. Hence, $\sum_{i=1}^{3}\left|L_{i} \cap W\right| \leq|W|+3$ since $|M| \leq 3$ by (2.4.9)(c).

(b) By $(2.4 .14)(\mathrm{a}),(2.4 .14)(\mathrm{e})$ and $(2.4 .15)(\mathrm{a})$, we have the following inequality:

$$
3 k=\sum_{i=1}^{3}\left|L_{i}\right| \leq \sum_{i=1}^{3}\left(\left|A_{i}\right|+\left|L_{i} \cap W\right|\right) \leq 3(k+1-|W|)+|W|+3=3 k+6-2|W| .
$$


Hence, $|W| \leq 3$. And if $|W|=3$ then $M=W$ and $\left|A_{i}\right|=k+1-|W|$.

2.4.16 We claim that, for $1 \leq j \leq n$, if $\left|W \cup X_{j}\right|<(k+2)$ then $X_{j}=Y_{j}$.

Suppose that $X_{j} \neq Y_{j}$. First we claim that $V(G)-Y_{j}-W$ is not empty. Since $\mid W \cup$ $X_{j} \mid \leq k+2$ and $\left|L_{1} \cup L_{2} \cup L_{3}\right|=3 k-|M| \geq 3 k-3 \geq k+3$ (by (2.4.9)(b) and (2.4.9)(c)), $\left(L_{1} \cup L_{2} \cup L_{3}\right)-\left(W \cup X_{j}\right) \neq \emptyset$. Hence $V(G)-Y_{j}-W$ which contains $\left(L_{1} \cup L_{2} \cup L_{3}\right)-\left(W \cup X_{j}\right)$ is not empty.

Note that $G$ is $(k+2)$-connected and by $(2.4 .8)(\mathrm{b}), W \cup X_{j}$ is a vertex-cut separating $Y_{j}-X_{j}$ and $V(G)-Y_{j}-W$ neither of which is empty. It follows that $\left|W \cup X_{j}\right| \geq(k+2)$, as required.

2.4.17 We claim that, for $1 \leq j \leq n$, if $\left|X_{j}\right|<3$ then $X_{j}=Y_{j}$.

By (2.4.16), it is obvious that $X_{j}=Y_{j}$ if $\left|X_{j}\right|<3$ since $k \geq 4$ (by (2.4.3)) and $|W| \leq 3$ (by $(2.4 .15))$.

2.4.18 Let $Z=\left(X_{1} \cup \cdots \cup X_{n}\right)-\left(L_{1} \cup L_{2} \cup L_{3}\right)$

\subsubsection{Some vertex-cuts of $G$}

Suppose that $X_{i} \cap L_{j} \neq \emptyset$ for some $i \in\{1,2, \cdots, n\}, j \in\{1,2,3\}$. By (2.4.8)(c), (2.4.14)(a) and (2.4.14)(d), any path joining $X_{i} \cap L_{j}$ and $L_{1} \cup L_{2} \cup L_{3}-W-L_{j}$ must use a vertex of $W$ or $Z$ or $X_{i} \Delta L_{j}$. Therefore, $\left(X_{i} \Delta L_{j}\right) \cup W \cup Z$ is a cutset of $G$ separating $X_{i} \cap L_{j}$ from $L_{1} \cup L_{2} \cup L_{3}-W-L_{j}$.

2.4.20 We claim that $\left|X_{i}\right| \geq 3$ for $1 \leq i \leq n$.

This claim is to be proved in several steps in this subsection.

(a) First we show that, for $1 \leq i \leq 3,1 \leq j \leq n$, if $\left|X_{j}\right|=1$, then $A_{i} \cap X_{j}=\emptyset$. 
Suppose $A_{1} \cap X_{j} \neq \emptyset$. Let $X_{j}=\{v\}$ and $N=N_{G}(v)$. Since $\mathrm{G}$ is $(k+2)$-connected, $|N| \geq k+2$. Hence $|N-W| \geq k+2-|W|$. Note that $\left|A_{1}\right| \leq k+1-|W|$ by (2.4.14)(e), this implies $N-A_{1}-W \neq \emptyset$. Take a vertex $x \in N-A_{1}-W$. Since $\left|X_{j}\right|=1$, we have $X_{j}=Y_{j}=\{v\}$ by (2.4.17). Note that $x v \in E(G), x$ is in $A_{1}$ by the definition of $A_{1}$, a contradiction. Hence $A_{1} \cap X_{j}=\emptyset$.

(b) Second we show that, for $1 \leq i \leq 3,1 \leq j \leq n$, if $\left|X_{j}\right|=1$, then $A_{i} \cap N_{G}\left(X_{j}\right)=\emptyset$.

Suppose that $\left|X_{1}\right|=1$ and $x \in A_{1} \cap N_{G}\left(X_{1}\right)$. Hence, by (2.4.14)(b), $x \in X_{i}$ for some $i \neq 1$. Since $\left|X_{1}\right|=1$, by the definition of $A_{1}$ (defined in (2.4.13)), $X_{1} \subseteq A_{1}$. This contradicts $(2.4 .20)(\mathrm{a})$ since $\left|X_{1}\right|=1$.

(c) Since $\left|X_{i}\right|$ is odd for each $i$ (by (2.4.11)), let $m$ be an integer such that $m \leq n$ with $\left|X_{i}\right|=1$ for $1 \leq i \leq m \leq n$ and $\left|X_{j}\right| \geq 3$ for $m<j \leq n$.

By the definition of $A_{i}$ and (2.4.9), we have

$$
\sum_{i=1}^{3}\left|A_{i}\right| \geq\left|L_{1} \cup L_{2} \cup L_{3}\right|-|W|=3 k-|M|-|W|
$$

Also, by $(2.4 .8)(\mathrm{a})$,

$$
\sum_{m<j \leq n}\left|X_{j}\right| \leq 3 \sum_{m<j \leq n}\left\lfloor\frac{1}{2}\left|X_{j}\right|\right\rfloor \leq 3 \sum_{1 \leq j \leq n}\left\lfloor\frac{1}{2}\left|X_{j}\right|\right\rfloor \leq 3(k+1-|W|)
$$

Assume $X=X_{1} \cup X_{2} \cup \cdots \cup X_{m}$ and $N=N_{G}(X)-X$. Then we can get the followings.

(i) $N \subseteq W \cup X_{m+1} \cup \cdots \cup X_{n}$ by (2.4.8)(b) and (2.4.17).

(ii) $N \cap A_{1}=N \cap A_{2}=N \cap A_{3}=\emptyset$ by $(2.4 .20)$ (b).

(iii) $|N| \geq k+2$ since $N$ separates $X$ from $A_{1} \cup A_{2} \cup A_{3}$ (by (2.4.20)(a) and (2.4.20)(b)) and $G$ is $(\mathrm{k}+2)$-connected.

Hence, we have 


$$
|N|+\left|A_{1}\right|+\left|A_{2}\right|+\left|A_{3}\right| \leq|W|+\sum_{i=m+1}^{n}\left|X_{i}\right|
$$

By (iii), (I), (II), and (III), we have

$$
\begin{gathered}
(k+2)+(3 k-|M|-|W|) \leq|W|+3(k+1-|W|) \\
=3 k+3-2|W| .
\end{gathered}
$$

Hence,

$$
|W| \leq 1+|M|-k
$$

By $(2.4 .9)(\mathrm{a})$,

$$
|W| \leq 1+|W|-k
$$

That is,

$$
k \leq 1
$$

This contradicts $k \geq 4(2.4 .3)$ and completes the proof of (2.4.20).

2.4.21 We prove some inequalities for $|Z|$.

(i)

$$
|Z| \leq 3 k+3-3|W|-\left|L_{1} \cup L_{2} \cup L_{3}-W\right|,
$$

and the equality holds if and only if $\left|X_{j}\right|=3$ for every $j \in\{1,2, \cdots, n\}$. 
(ii)

$$
|Z| \leq 3+|M|-2|W|
$$

and the equality holds if and only if $\left|X_{j}\right|=3$ for every $j \in\{1,2, \cdots, n\}$ and $W \subseteq L_{1} \cup L_{2} \cup L_{3}$.

Let $s=|Z|$. Then, by (2.4.18),

$$
\left|X_{1} \cup \cdots \cup X_{n}\right|=s+\left|L_{1} \cup L_{2} \cup L_{3}-W\right|
$$

But, by (2.4.20), $\left|X_{j}\right| \leq 3\left\lfloor\frac{1}{2}\left|X_{j}\right|\right\rfloor$ for $1 \leq j \leq n$, and therefore

$$
3 \sum_{1 \leq j \leq n}\left\lfloor\frac{1}{2}\left|X_{j}\right|\right\rfloor \geq \sum_{1 \leq j \leq n}\left|X_{j}\right|=s+\left|L_{1} \cup L_{2} \cup L_{3}-W\right|,
$$

with equality if and only if $\left|X_{j}\right|=3$ for any $j \in\{1,2, \cdots, n\}$. By (2.4.8)(a), we have

$$
3(k+1-|W|) \geq s+\left|L_{1} \cup L_{2} \cup L_{3}-W\right| .
$$

That is,

$$
s \leq 3 k+3-3|W|-\left|L_{1} \cup L_{2} \cup L_{3}-W\right|,
$$

and the equality holds if and only if $\left|X_{j}\right|=3$ for any $j \in\{1,2, \cdots, n\}$. That completes the proof of $(2.4 .21)(\mathrm{i})$.

Note that, by $(2.4 .9)(\mathrm{b})$, we have

$$
\left|L_{1} \cup L_{2} \cup L_{3}-W\right| \geq\left|L_{1} \cup L_{2} \cup L_{3}\right|-|W|=3 k-|M|-|W|,
$$

and the equality holds if and only if $W \subseteq L_{1} \cup L_{2} \cup L_{3}$. Hence, by (2.4.21)(i),

$$
\begin{aligned}
& s \leq 3 k+3-3|W|-\left|L_{1} \cup L_{2} \cup L_{3}-W\right| \leq 3 k+3-3|W|-(3 k-|M|-|W|) \\
& =3+|M|-2|W|,
\end{aligned}
$$

and the equality holds if and only if $W \subseteq L_{1} \cup L_{2} \cup L_{3}$ and $\left|X_{j}\right|=3$ for every $j \in\{1,2, \cdots, n\}$. This completes the proof of (2.4.21)(ii).

2.4 .22 (i) $\left|A_{i} \cap X_{j}\right|<\frac{1}{2}\left|X_{j}\right|$ for $1 \leq j \leq n$ and $1 \leq i \leq 3$. 
Suppose that $\left|A_{1} \cap X_{1}\right| \geq \frac{1}{2}\left|X_{1}\right|$. Since $\left|X_{1}\right| \geq 3$ by (2.4.20), there exists a vertex $v \in A_{1} \cap X_{1}$. Since $\left|L_{2} \cup L_{3}-W\right| \geq\left|L_{2} \cup L_{3}\right|-|W| \geq k+2-|W|$ by (2.4.9)(d), and $G-W$ is $(k+2-|W|)$ connected, there are $(k+2-|W|)$ paths of $G-W$ between $A_{1}$ and $L_{2} \cup L_{3}-W$, disjoint except possibly for $v$. Choose them with no internal vertex in $A_{1}$. By $(2.4 .14)(\mathrm{d})$, each has at least two vertices in $X_{j}$ for some $j$, but at most $\left\lfloor\frac{1}{2}\left|X_{j}\right|\right\rfloor$ of them have two vertices in $X_{j}$ for each $j \neq 1$. Note that by $(2.4 .8)(\mathrm{a})$, we have

$$
\sum_{2 \leq j \leq n}\left\lfloor\frac{1}{2}\left|X_{j}\right|\right\rfloor \leq k+1-|W|-\left\lfloor\frac{1}{2}\left|X_{1}\right|\right\rfloor .
$$

Thus, at least $1+\left\lfloor\frac{1}{2}\left|X_{1}\right|\right\rfloor$ of them have two vertices in $X_{1}$. But each has only one vertex in $A_{1}$, and so has a vertex in $X_{1}$ which does not belong to $A_{1}$, and all these vertices in $X_{1}-A_{1}$ are different. Hence $\left|X_{1}-A_{1}\right| \geq 1+\left\lfloor\frac{1}{2}\left|X_{1}\right|\right\rfloor$, a contradiction.

$$
\left|L_{i} \cap X_{j}\right|<\frac{1}{2}\left|X_{j}\right| \text { for } 1 \leq j \leq n \text { and } 1 \leq i \leq 3 \text { by (2.4.14)(a) and (2.4.22)(i). }
$$

2.4.23 We eliminate the case of $k=4$ and $|W|=3$.

In this case, by $(2.4 .12)(\mathrm{a}), n \geq k-2=2$.

Moreover, by (2.4.8)(a), we have

$$
|W|+\sum_{1 \leq i \leq n}\left\lfloor\frac{1}{2}\left|X_{i}\right|\right\rfloor \leq k+1=5 .
$$

That is,

$$
\sum_{1 \leq i \leq n}\left\lfloor\frac{1}{2}\left|X_{i}\right|\right\rfloor \leq 2
$$

We have $n=2$ and $\left|X_{1}\right|=\left|X_{2}\right|=3$ (by (2.4.20)). Hence, $Z=\emptyset$ by $(2.4 .12)(\mathrm{b})$.

Next we claim that $\left|L_{i} \cap X_{j}\right| \neq 0$ for $i \in\{1,2,3\}$ and $j \in\{1,2\}$. For otherwise, we may assume that $L_{1} \cap X_{1}=\emptyset$. Then by (2.4.22)(ii), $\left|L_{2} \cap X_{1}\right| \leq 1$ and $\left|L_{3} \cap X_{1}\right| \leq 1$. This implies that $Z \supseteq X_{1}-\left(L_{1} \cup L_{2} \cup L_{3}\right)=X_{1}-L_{2} \cup L_{3} \neq \emptyset$. This contradicts that $Z=\emptyset$.

Therefore, by (2.4.22)(ii), we have $\left|L_{i} \cap X_{j}\right|=1$ for $i \in\{1,2,3\}$ and $j \in\{1,2\}$. And by (2.4.12) (b), we have $W=M$ and $W \cup X_{1} \cup X_{2}=L_{1} \cup L_{2} \cup L_{3}$. Hence, $\left|W \cap L_{i}\right|=2$ for $i \in\{1,2,3\}$. 
Next, we will apply Lemma (2.3.5) to find a 6-cluster in $G$ traversing $L_{1} \cup L_{2} \cup L_{3}$.

Let $X_{1}=\left\{x_{1}, x_{2}, x_{3}\right\}, X_{2}=\left\{y_{1}, y_{2}, y_{3}\right\}, W=\left\{z_{1}, z_{2}, z_{3}\right\}$, let $\left\{x_{i}, y_{i}\right\} \subseteq L_{i}$ for $i \in\{1,2,3\}$, and $z_{1} \in L_{2} \cap L_{3}, z_{2} \in L_{1} \cap L_{3}, z_{3} \in L_{1} \cap L_{2}$ (by (2.4.2)).

Hence, we get the description of graph $G$ as in Lemma (2.3.5): $x_{i}, y_{i}, z_{i}(1 \leq i \leq 3)$ are distinct vertices of 6 -connected graph $G$, and $L_{1}=\left\{x_{1}, y_{1}, z_{2}, z_{3}\right\}, L_{2}=\left\{x_{2}, y_{2}, z_{3}, z_{1}\right\}$, $L_{3}=\left\{x_{3}, y_{3}, z_{1}, z_{2}\right\}$ are 4-cliques, and there is a partition $Y_{1}, Y_{2}$ of $V(G)-\left\{z_{1}, z_{2}, z_{3}\right\}$ with $X_{1} \subseteq Y_{1}, X_{2} \subseteq Y_{2}$, and $x_{i} y_{i}(1 \leq i \leq 3)$ are the only edges of $G$ with one end in $Y_{1}$ and the other in $Y_{2}$.

Therefore by Lemma (2.3.5), $G$ has a 6 -cluster traversing $L_{1} \cup L_{2} \cup L_{3}$. This is a contradiction.

2.4.24 We claim that, for $1 \leq j \leq n$, if $\left|X_{j}\right|=3$ then $X_{j}=Y_{j}$.

By (2.4.15), $|W| \leq 3$. By (2.4.16), it is obvious that $X_{j}=Y_{j}$ if $k \geq 5$ or $|W|<3$. Hence, the only remaining case is $k=4$ and $|W|=3$, which was eliminated in (2.4.23).

2.4.25 (i) We claim that if $v \in A_{i} \cap X_{j}$ for some $i \in\{1,2,3\}$ and some $j \in\{1,2, \cdots, n\}$, then $d_{Y_{j}-A_{i}}(v) \geq 2$, and the equality holds if and only if $d_{G}(v)=k+2, W \cup A_{i} \subseteq N_{G}(v) \cup\{v\}$ and $\left|A_{i}\right|=k+1-|W|$.

By the definition of $A_{i}(2.4 .13)$, we have

$$
N_{G}(v)-\left(Y_{j}-A_{i}\right) \subseteq A_{i} \cup W-\{v\} .
$$

Since $G$ is $(k+2)$-connected and $\left|A_{i}\right| \leq k+1-|W|$ (by (2.4.14)(e)), we have:

$$
\left|N_{G}(v) \cap\left(Y_{j}-A_{i}\right)\right| \geq(k+2)-\left|A_{i} \cup W-\{v\}\right| \geq(k+2)-(k+1-|W|+|W|-1)=2
$$

and the equality holds if and only if $d(v)=k+2, W \cup A_{i} \subseteq N_{G}(v) \cup\{v\}$ and $\left|A_{i}\right|=k+1-|W|$.

(ii) We claim that if $v \in A_{i} \cap X_{j}$ and $\left|X_{j}\right|=3$ for some $i \in\{1,2,3\}$ and some $j \in\{1,2, \cdots, n\}$, then $d_{X_{j}}(v)=2, W \cup A_{i} \subseteq N_{G}(v) \cup\{v\}$ and $\left|A_{i}\right|=k+1-|W|$.

Note that $\left|X_{j}\right|=3$. By (2.4.24), we have $Y_{j}=X_{j}$, and therefore,

$$
d_{Y_{j}-A_{i}}(v)=d_{X_{j}-A_{i}}(v) \leq 2 .
$$


On the other hand, by (2.4.25)(i), we have $d_{Y_{j}-A_{i}}(v) \geq 2$. Hence, $d_{Y_{j}-A_{i}}(v)=2$. By (2.4.25)(i) again, we are done.

2.4.26 We claim that if $\left|X_{j}\right|=3$ for some $j$, then

(i) $\left|X_{j} \cap A_{i}\right|=1$ for each $i \in\{1,2,3\}$,

(ii) $\quad X_{j}$ induces a clique of $G$, and

(iii) $\quad W \subseteq N_{G}(v)$ for any $v \in X_{j}$.

Proof of (i): For otherwise, we may assume that $\left|X_{j} \cap A_{1}\right| \neq 1$. By (2.4.22)(i), $\left|X_{j} \cap A_{1}\right|=0$, $\left|A_{2} \cap X_{j}\right| \leq 1$ and $\left|A_{3} \cap X_{j}\right| \leq 1$. This implies that there exists $x \in X_{j}$, and $x \notin A_{1} \cup A_{2} \cup A_{3}$. Since $\left|X_{j}\right|=3$, we have $X_{j}=Y_{j}$ by (2.4.24), and by the definition of $A_{i}$ (2.4.13), we have $N_{G}(x) \subseteq W \cup(Z-\{x\}) \cup\left(X_{j}-\{x\}\right)$. Note that, by (2.4.21)(ii), (2.4.15), we have

$$
|W|+|Z-\{x\}|+\left|X_{j}-\{x\}\right| \leq|W|+(3+|M|-2|W|-1)+2=4+|M|-|W| .
$$

Note that $|M| \leq|W|$ by $(2.4 .9)$ (a). Hence, we have $\left|N_{G}(x)\right| \leq 4$. This contradicts that $G$ is $(k+2)$-connected where $k \geq 4$ (by $(2.4 .3))$.

Proof of (ii) and (iii). (ii) and (iii) are immediate corollaries of (2.4.26)(i) and (2.4.25)(ii).

2.4.27 We claim that there exists some $j \in\{1,2, \cdots, n\}$ such that $\left|X_{j}\right| \geq 5$.

By (2.4.20), we may assume $\left|X_{j}\right|=3$ for all $j \in\{1,2, \cdots, n\}$.

By (2.4.26)(i)-(ii), every vertex of $\left(L_{1}-W\right) \cap X_{j}$ (for all $j \in\{1,2, \cdots, n\}$ ) is adjacent to a vertex of $A_{2} \cap X_{j}$ and a vertex of $A_{3} \cap X_{j}$. By (2.4.26)(i) and (2.4.26)(iii), every vertex of $L_{1} \cap W$ is adjacent to some vertex of $A_{2}$ and some vertex of $A_{3}$, hence, $\wp=\left\{u_{1}, u_{2}, \cdots, u_{k}, A_{2}, A_{3}\right\}$ is a $(k+2)$-cluster that traverses $L_{1} \cup L_{2} \cup L_{3}$ where $\left\{u_{1}, u_{2}, \cdots, u_{k}\right\} \in L_{1}$. This is a contradiction.

2.4.28 We claim that $\left|X_{j}\right| \geq 5$ for every $j \in\{1,2, \cdots, n\}$.

For otherwise, by (2.4.20), we may assume $\left|X_{1}\right|=3$. By (2.4.26)(i), $\left|A_{i} \cap X_{1}\right|=1$ for each $i \in\{1,2,3\}$. Hence, by (2.4.25)(ii), $\left|A_{i}\right|=k+1-|W|$ for each $i \in\{1,2,3\}$. 
Furthermore, by $(2.4 .14)(\mathrm{b}),(2.4 .14)(\mathrm{c})$, we have

$|Z| \geq\left|A_{1}\right|+\left|A_{2}\right|+\left|A_{3}\right|-\left|L_{1} \cup L_{2} \cup L_{3}-W\right|=(3 k+3-3|W|)-\left|L_{1} \cup L_{2} \cup L_{3}-W\right|$.

However, by $(2.4 .21)(\mathrm{i})$, we have

$$
|Z|=3 k+3-3|W|-\left|L_{1} \cup L_{2} \cup L_{3}-W\right| .
$$

The equality of (2.4.21)(i) implies that $\left|X_{i}\right|=3$ for all $i \in\{1,2, \cdots, n\}$. This contradicts (2.4.27).

\subsubsection{We show some inequalities for $n$.}

By (2.4.28) and (2.4.8)(a),

$$
5 n \leq \sum_{1 \leq j \leq n}\left|X_{j}\right| \leq 2 *(k+1-|W|)+n=2 k+2+n-2|W|
$$

The inequality (IV) can be simplified as

$$
2 n \leq k+1-|W|
$$

Note that the equality of (IV) (and (V), as well) holds if and only if $\left|X_{i}\right|=5$ for every $i$.

2.4.30 We claim that $n=k-2$.

For otherwise, since $n \geq k-2$ by (2.4.12)(a), we may assume that $n \geq k-1$.

By (V), we have

$$
2 k-2 \leq 2 n \leq k+1-|W|
$$

That is,

$$
k \leq 3-|W| .
$$

Note that $k \geq 4$ by (2.4.3). This is a contradiction. 


\subsubsection{The final step of the proof.}

By (2.4.30), $n=k-2$. By (2.4.12)(b), we have

$$
W=M \text { and } L_{1} \cup L_{2} \cup L_{3}=W \cup X_{1} \cup \cdots \cup X_{n} .
$$

Hence,

$$
Z=\emptyset
$$

By (V) of (2.4.29), we have

$$
2 k-4=2 n \leq k+1-|W|
$$

That is,

$$
k \leq 5-|W|
$$

Note that $k \geq 4$ by (2.4.3). Therefore, there are only two cases: $k=5$ and $k=4$ (by (VII) and (VIII)).

Case 1: $k=5$. In this case, $|W|=0$. By (2.4.30), $n=k-2=3$. The equality of (V) of (2.4.29) implies that

$$
\left|X_{1}\right|=\left|X_{2}\right|=\left|X_{3}\right|=5
$$

By (2.4.22)(ii), without loss of generality, we assume $\left|L_{1} \cap X_{1}\right|=2$. By $(2.4 .19),\left(X_{1} \Delta L_{1}\right)$ is a vertex-cut of order at most 6 since $Z=\emptyset$ and $W=\emptyset$. This contradicts that $G$ is $(k+2)$ connected where $k=5$.

Case 2: $k=4$. In this case, $n=k-2=2$ (by (2.4.30)). There are two subcases: $|W|=1$ and $|W|=0$ (by VIII).

Subcase 1: $|W|=1$. The equality of (V) of (2.4.29) implies that

$$
\left|X_{1}\right|=\left|X_{2}\right|=5 \text {. }
$$

Without loss of generality, we assume $W \subseteq L_{1}$ and $\left|L_{1} \cap X_{1}\right|=2$. By (2.4.19), $\left(X_{1} \Delta L_{1}\right)$ is a vertex-cut of order at most 5 since $Z=\emptyset$ and $W \subseteq L_{1}$. This contradicts that $G$ is $(k+2)$-connected where $k=4$.

Subcase 2: $|W|=0$.

Since $Z=\emptyset$ by (VII), we have

$$
\sum_{j=1}^{n}\left|X_{j}\right|=\left|L_{1} \cup L_{2} \cup L_{3}\right|=3 k=12 .
$$


Therefore, the only possibility in this subcase is that $\left|X_{1}\right|=5$ and $\left|X_{2}\right|=7$ (by (2.4.11) and (2.4.28)).

Without loss of generality, we assume $\left|L_{1} \cap X_{1}\right|=2$. By (2.4.19), $\left(X_{1} \Delta L_{1}\right)$ is a vertex-cut of order at most 5 since $Z=\emptyset$ and $W=\emptyset$. This contradicts that $G$ is $(k+2)$-connected where $k=4$.

This completes the proof of main theorem (Theorem 2.1.12).

\subsection{Proof of Lemma 2.3.5}

The following two lemmas will be useful in the proof of Lemma 2.3.5.

Lemma 2.5.1 (Whitney [8]) Any two planar embeddings of a 3-connected graph are equivalent.

Let $G=(V, E)$ is a graph and $A \subseteq V(G)$, we denote the set of vertices on $V(G)-A$ which are adjacent to some vertex in $A$ by $\Upsilon(A)$. That is $\Upsilon(A)=N(A)-A$.

Lemma 2.5.2 (Seymour [28], Thomassen [32]) Let $s_{1}, t_{1}, s_{2}, t_{2}$ be distinct vertices of a graph $G=(V, E)$. Then just one of the following is true:

(i) there are paths joining $s_{1}$ to $t_{1}$ and $s_{2}$ to $t_{2}$ respectively, vertex-disjoint.

(ii) for some $k \geq 0$ there are pairwise disjoint sets $A_{1}, \cdots, A_{k} \subseteq V(G)-\left\{s_{1}, s_{2}, t_{1}, t_{2}\right\}$ such that

(a) for $i \neq j, \Upsilon\left(A_{i}\right) \cap A_{j}=\emptyset$,

(b) for $1 \leq i \leq k,\left|\Upsilon\left(A_{i}\right)\right| \leq 3$,

(c) if $\widetilde{G}$ is the graph obtained from $G$ by (for each i) deleting $A_{i}$ and adding new edges joining every pair of distinct vertices in $\Upsilon\left(A_{i}\right)$, and also for $j=1,2$ adding an edge $e_{j}$ joining 
$s_{j}$ to $t_{j}$, then $\widetilde{G}$ may be drawn in the plane with no pairs of edges crossing except $e_{1}$, $e_{2}$ which cross once.

Proof of Lemma 2.3.5: Let $G$ be a counterexample to the lemma with least number of vertices. Let $W=\left\{z_{1}, z_{2}, z_{3}\right\}, X_{1}=\left\{x_{1}, x_{2}, x_{3}\right\}, X_{2}=\left\{y_{1}, y_{2}, y_{3}\right\}$. Let $\Im_{i j}=\left\{z_{i}\right\} \cup X_{j}$ and $G_{i j}$ be the subgraph induced by $\left\{z_{i}\right\} \cup Y_{j}$ where $1 \leq i \leq 3$ and $1 \leq j \leq 2$. Let $f_{1}, f_{2}, f_{3}$ be the edges with ends $z_{2} z_{3}, z_{3} z_{1}$, and $z_{1} z_{2}$ respectively.

2.5.1 We claim that $G-\left\{f_{1}, f_{2}, f_{3}\right\}$ is not planar.

Since $G$ is 6-connected, $|E(G)| \geq 3|V(G)|$. Therefore $\left|E\left(G-\left\{f_{1}, f_{2}, f_{3}\right\}\right)\right| \geq 3|V(G)|-3$. Note that a planar graph with $n \geq 3$ vertices has at most $3 n-6$ edges. Hence $G-\left\{f_{1}, f_{2}, f_{3}\right\}$ is not planar.

\subsection{2}

The strategy of the proof is to prove that $G^{\prime}=G-\left\{f_{1}, f_{2}, f_{3}\right\}$ is planar (hence contradicts 2.5.1). The planarity of $G^{\prime}$ is yielded by showing that each $G_{i}=G^{\prime}\left[W \cup Y_{i}\right](1 \leq i \leq 2)$ has a planar embedding with vertices $z_{1}, x_{2}, z_{3}, x_{1}, z_{2}, x_{3}$ for $i=1$ (or $z_{1}, y_{2}, z_{3}, y_{1}, z_{2}, y_{3}$ for $i=2$, respectively) around its exterior face (Lemma 2.5.2 is applied here). And the planar embedding of $G_{i}$ is constructed from the unique embedding of the 3 -connected graph $H_{i}=Y_{i} \cup E_{i}(1 \leq i \leq 2)$ where $E_{1}=\left\{x_{1} x_{2}, x_{1} x_{3}, x_{2} x_{3}\right\}$, and $E_{2}=\left\{y_{1} y_{2}, y_{1} y_{3}, y_{2} y_{3}\right\}$, and planar embeddings of other subgraphs of $G_{i} \cup E_{i}$.

2.5.3 We claim that $\left|Y_{1}\right|,\left|Y_{2}\right| \geq 4$.

For otherwise, we may assume $\left|Y_{1}\right|=3$. That is, $Y_{1}=X_{1}$. Since $G$ is 6-connected, $d_{G}\left(x_{1}\right) \geq 6$. Hence $W \cup\left\{y_{1}, x_{2}, x_{3}\right\} \subseteq N_{G}\left(x_{1}\right)$. With the similar argument, we have $W \cup$ $\left\{y_{3}, x_{1}, x_{2}\right\} \subseteq N_{G}\left(x_{3}\right)$ and $W \cup\left\{y_{2}, x_{1}, x_{3}\right\} \subseteq N_{G}\left(x_{2}\right)$. Therefore, $\wp=\left\{\left\{x_{1}\right\},\left\{x_{2}\right\},\left\{x_{3}\right\},\left\{z_{1}\right\}\right.$, $\left.\left\{z_{2}\right\},\left\{z_{3}\right\}\right\}$ is a 6 -cluster traversing $\left\{x_{1}, y_{1}, z_{1}, x_{2}, y_{2}, z_{2}, x_{3}, y_{3}, z_{3}\right\}$, a contradiction.

2.5.4 We claim that the subgraph induced by $Y_{i}$ is connected for $i=1,2$. 
For otherwise, we assume that the subgraph induced by $Y_{1}$ is not connected. Let $D$ be a component not containing $x_{1}$, then $\left\{x_{2}, x_{3}, z_{1}, z_{2}, z_{3}\right\}$ is a 5-cutset separating $D$ and $Y_{2}$, it contradicts that $G$ is 6 -connected.

2.5.5 We claim that there is no 4-cluster in $G_{i j}$ traversing $\Im_{i j}$ where $1 \leq i \leq 3,1 \leq j \leq 2$.

For otherwise, we may assume that there is a 4-cluster $\wp_{1}$ in $G_{11}$ traversing $\Im_{11}$. Let $\wp=\wp_{1} \cup\left\{\left\{z_{2}, z_{3}\right\},\left\{Y_{2}\right\}\right\}$, then $\wp$ is a 6 -cluster in $G$ traversing $\left\{x_{1}, y_{1}, z_{1}, x_{2}, y_{2}, z_{2}, x_{3}, y_{3}, z_{3}\right\}$, a contradiction.

2.5.6 We claim that both $X_{1}$ and $X_{2}$ are independent sets of $G$.

For otherwise, without loss of generality, we assume that $x_{1} x_{2} \in E(G)$. There is a vertex $x \in Y_{1}-X_{1}$ by (2.5.3) and there are 4 internally disjoint paths $P_{x_{1}}, P_{x_{2}}, P_{x_{3}}, P_{z_{3}}$ of $G-\left\{z_{1}, z_{2}\right\}$ since $G-\left\{z_{1}, z_{2}\right\}$ is 4 -connected where $P_{u}$ is the path joining $x$ and $u \in\left\{x_{1}, x_{2}, x_{3}, z_{3}\right\}$.

Now since $x_{i} y_{i}(1 \leq i \leq 3)$ are the only edges of $G$ with one end in $Y_{1}$ and the other in $Y_{2}$, we have $P_{u} \cap Y_{2}=\emptyset$ where $u \in\left\{x_{1}, x_{2}, x_{3}, z_{3}\right\}$. Hence $\wp=\left\{P_{x_{1}}-x, P_{x_{2}}-x, P_{z_{3}}-x, P_{x_{3}}\right.$ is a 4-cluster in $G_{31}$ traversing $\Im_{31}$. This contradicts (2.5.5).

2.5.7 We claim that there are no two vertex-disjoint paths in $G_{11}$ joining $z_{1}$ to $x_{1}$ and $x_{2}$ to $x_{3}$ respectively.

For otherwise, let $P_{1}$ and $P_{2}$ be the paths joining $z_{1}$ to $x_{1}$ and $x_{2}$ to $x_{3}$ respectively. Let $A_{1}$ and $A_{2}$ be disjoint fragments of the subgraph induced by $Y_{1}$ with $P_{1}-\left\{z_{1}\right\} \subseteq A_{1}$, $P_{2} \subseteq A_{2}$, and $A_{1} \cup A_{2}$ maximal. Since the subgraph induced by $Y_{1}$ is connected by (2.5.4), $A_{1}$ and $A_{2}$ are adjacent. Therefore $\wp=\left\{\left\{z_{1}\right\},\left\{z_{2}\right\},\left\{z_{3}\right\}, A_{1}, A_{2}, Y_{2}\right\}$ is a 6 -cluster traversing $\left\{x_{1}, y_{1}, z_{1}, x_{2}, y_{2}, z_{2}, x_{3}, y_{3}, z_{3}\right\}$, a contradiction.

2.5.8 (i) We claim that $G_{11}$ can be drawn in a plane so that every vertex in $\Im_{11}$ is incident with the infinite region and $z_{1}, x_{2}, x_{1}, x_{3}$ are around its exterior face in this order, and this embedding is denoted by $\pi_{11}$. 
By Lemma 2.5.2 and (2.5.7), there exists some $k \geq 0$ and there are pairwise disjoint sets $A_{1}, \cdots, A_{k} \subseteq V(G)-\left\{z_{1}, x_{2}, x_{1}, x_{3}\right\}$ such that

(a) for $i \neq j, \Upsilon\left(A_{i}\right) \cap A_{j}=\emptyset$,

(b) for $1 \leq i \leq k,\left|\Upsilon\left(A_{i}\right)\right| \leq 3$,

(c) if $\widetilde{G_{11}}$ is the graph obtained from $G_{11}$ by deleting $A_{i}$ and adding new edges joining every pair of distinct vertices in $\Upsilon\left(A_{i}\right)$, and adding an edge $e_{1}$ joining $z_{1}$ to $x_{1}$ and an edge $e_{2}$ joining $x_{2}$ to $x_{3}$, then $\widetilde{G_{11}}$ may be drawn in the plane with no pairs of edges crossing except $e_{1}, e_{2}$ which cross once.

We claim that $k=0$. For otherwise, $\Upsilon\left(A_{k}\right) \cup\left\{z_{2}, z_{3}\right\}$ is a cut set of order at most 5 separating $A_{k}$ and $Y_{2}$, it contradicts that $G$ is 6 -connected.

Hence we have $G_{11}=\widetilde{G_{11}}-\left\{e_{1}, e_{2}\right\}$, and result follows.

With the similar argument, we have

(ii) $G_{21}$ can be drawn in a plane so that $z_{2}, x_{1}, x_{2}, x_{3}$ are around its exterior face in this order, and this embedding is denoted by $\pi_{21}$.

(iii) $G_{31}$ can be drawn in a plane so that $z_{3}, x_{1}, x_{3}, x_{2}$ are around its exterior face in this order, and this embedding is denoted by $\pi_{31}$.

2.5.9 Let $G_{i}$ be the graph obtained from the subgraph induced by $W \cup Y_{i}$ and deleting edges $f_{1}$, $f_{2}, f_{3}$ for $1 \leq i \leq 2$, and let $G_{1}^{+}$be the graph obtained from $G_{1}$ by adding edges $x_{1} x_{2}, x_{1} x_{3}$, $x_{2} x_{3}$, let $G_{2}^{+}$be the graph obtained from $G_{2}$ by adding edges $y_{1} y_{2}, y_{1} y_{3}, y_{2} y_{3}$.

In next a few subsections, we are to show that $G_{1}^{+}$has a planar embedding such that the triangle $x_{1} x_{2} x_{3} x_{1}$ is a facial circuit.

\subsubsection{0}

(i) Let $H_{h 1}$ be the graph obtained from $G_{1}^{+}$by deleting $z_{i}$ and $z_{j}$ where $\{h, i, j\}=\{1,2,3\}$. Obviously $H_{h 1}$ is also the graph obtained from $G_{h 1}$ by adding edges $x_{1} x_{2}, x_{1} x_{3}, x_{2} x_{3}$. By (2.5.8), $G_{h 1}$ has an embedding $\pi_{h 1}$ with $\left\{x_{i}, x_{h}, x_{j}, z_{h}\right\}$ in its exterior face. Hence $\pi_{h 1}$ can also 
be considered as an embedding of $H_{h 1}$ with the triangle $x_{1} x_{2} x_{3}$ as the exterior face. We denote this embedded graph by $\pi_{h 1}\left(H_{h 1}\right)$.

(ii) Let $H_{1}$ be the graphs obtained from the subgraph induced by $Y_{1}$ by adding edges $x_{1} x_{2}, x_{1} x_{3}$, $x_{2} x_{3}$. Obviously, $H_{1}$ can also be obtained from $H_{h 1}$ by deleting $z_{h}$ for $h \in\{1,2,3\}$. Hence $\pi_{h 1}$ can also be considered as an embedding of $H_{1}$, and we denote this embedded graph by $\pi_{h 1}\left(H_{1}\right)$.

2.5.11 We claim that $H_{1}$ is a 3-connected planar graph.

Planarity is an immediate conclusion from (2.5.10) (ii). Next we will show that $H_{1}$ is 3-connected.

For otherwise, we assume that $H_{1}$ is not 3-connected. Let $A$ be a cut set of order less than 3. Since $\left\{x_{1}, x_{2}, x_{3}\right\}$ induces a clique in the graph $H_{1}$, let $\mathrm{D}$ be a component of $H_{1}-A$ with $x_{i} \notin D$ for every $i \in\{1,2,3\}$. Then $A \cup\left\{z_{1}, z_{2}, z_{3}\right\}$ is a cutset of $G$ of order at most 5 separating $D$ and $Y_{2}$, it is a contradiction.

2.5.12 By lemma (2.5.1), $H_{1}$ has only one embedding $\pi_{0}$. That is, $\pi_{0}\left(H_{1}\right)=\pi_{h 1}\left(H_{1}\right)$ for each $h \in\{1,2,3\}$.

2.5.13 (i) We claim that $G_{1}^{+}$has a planar embedding such that the triangle $x_{1} x_{2} x_{3} x_{1}$ is a facial circuit.

Let $C_{i j}$ be the facial circuit of the embedded graph $\pi_{0}\left(H_{1}\right)$ containing the edge $x_{i} x_{j}$ other than the triangle $x_{1} x_{2} x_{3} x_{1}$. Let $F_{i j}$ be the face of $\pi_{0}\left(H_{1}\right)$ bounded by $C_{i j}$ where $1 \leq i<j \leq 3$.

By $(2.5 .12), \pi_{0}\left(H_{1}\right)=\pi_{11}\left(H_{1}\right)$, and by $(2.5 .10)$ (ii), $\pi_{11}\left(H_{1}\right)$ is obtained from $\pi_{11}\left(H_{11}\right)$ by deleting the vertex $z_{1}$, hence $z_{1}$ must be inside the face $F_{23}$ of $\pi_{0}\left(H_{1}\right)$, and all neighborhoods of $z_{1}$ must be in the facial circuit $C_{23}$. Similarly, for each $\{h, i, j\}=\{1,2,3\}$, all neighborhoods of $z_{h}$ must be in the facial circuit $C_{i j}$.

Note that $F_{i j}$ 's are distinct for $1 \leq i<j \leq 3$ since $H_{1}$ is 3 -connected by (2.5.11). Now we can get a planar embedding of $G_{1}^{+}$by adding the vertex $z_{h}$ and edges $z_{h} u$ into the face $F_{i j}$ where $u \in N_{G}\left(z_{h}\right) \cap Y_{1}$.

With the similar argument, we have 
(ii) $G_{2}^{+}$has a planar embedding such that the triangle $y_{1} y_{2} y_{3} y_{1}$ is a facial circuit.

\subsubsection{The final step of the proof.}

By (2.5.13) and (2.5.9), $G_{i}$ is planar with triangle $z_{1} z_{2} z_{3}$ as the exterior face.

Now we identify $z_{i}$ of $G_{1}$ to $z_{i}$ of $G_{2}$ where $1 \leq i \leq 3$, and join $x_{i}$ of $G_{1}$ to $y_{i}$ of $G_{2}$, we get planar graph $G-\left\{f_{1}, f_{2}, f_{3}\right\}$, it contradicts (2.5.1).

This completes the proof of this lemma. 


\section{Chapter 3}

\section{Hadwiger's Conjecture for Claw-free Graphs}

\subsection{Introduction}

Claw-free graphs have been a subject of interest to many researchers in recent years. In this chapter, we give some results on claw-free graphs.

If $H$ is a graph, then we say that $G$ is $H$-free if $G$ does not contain a copy of $H$ as an induced subgraph. By the claw, we mean the complete bipartite graph $K_{1,3}$. Thus $G$ is said to be claw-free if it does not contain an induced subgraph that is isomorphic to claw.

If $G$ is a graph, then the line graph of $G$, usually denoted by $L(G)$, is obtained by associating one vertex to each edge of $G$, and two vertices of $L(G)$ being joined by an edge if and only if the corresponding edges in $G$ are adjacent. Beineke [4, 5] and Robertson [23] (see also page 74 of [12]) independently gave a characterization of line graphs in terms of forbidden induced subgraphs and the claw is one (among nine) of those subgraphs. Thus every line graph is claw-free.

As stated in Chapter 2, Hadwiger's Conjecture is as follows:

Conjecture 3.1.1 (Hadwiger [10]) For all $k \geq 1$, every $k$-chromatic graph has the complete 
graph $K_{k}$ as a minor.

Our motivation is the following proposition.

Proposition 3.1.2 Hadwiger's Conjecture is true for line graphs of simple graphs.

Note that every line graph is claw-free, we may ask the following question.

Question 3.1.3 Is Hadwiger's Conjecture true for claw-free graphs?

Hadwiger's Conjecture is open for $k \geq 7$ as stated in Chapter 2, we may focus on the special case $k=7$ of Hadwiger's Conjecture, and we may ask the following questiones.

Question 3.1.4 Let $G$ be a claw-free 7-chromatic graph, does $G$ contain the complete graph $K_{7}$ as a minor?

Question 3.1.5 Let $G$ be a claw-free 6-connected 7-chromatic graph, does $G$ contain the complete graph $K_{7}$ as a minor?

In this chapter, we prove the following result,

Theorem 3.1.6 Let $G$ be a 6-connected claw-free graph. If $\delta(G) \geq 7$ and $G$ contains three disjoint 5-cliques, say $L_{1}, L_{2}, L_{3}$, then $G$ contains a $K_{7}$ as a minor.

We hope our result would be used to prove the Question 3.1.5.

\subsection{Proof of the main theorem}

Prove by way of contradiction. Assume $G$ doesn't contain a $K_{7}$ as a minor, and the following assertion is obvious by Menger's Theorem. 
3.2.1 The graph $G$ contains no clique of order 6 .

3.2.2 A path $P$ of $G$ with ends $u, v$ is said to be good if there exist distinct $i, j$ with $1 \leq i, j \leq 3$ such that $u \in L_{i}$ and $v \in L_{j}$.

3.2.3 We claim that there don't exist 7 mutually disjoint good paths in $G$.

Let $P_{1}, P_{2}, \cdots, P_{7}$ be a set of disjoint good paths of $G$. Let $G^{\prime}$ be the graph obtained by contracting $P_{i}$ to a new vertex $v_{i}$ for all $i \in\{1,2, \cdots, 7\}$. The subgraph $Q$ of $G^{\prime}$ induced by $v_{i}$ $(1 \leq i \leq 7)$ is a $K_{7}$-clique and corresponds to a $K_{7}$-minor in $G$.

By Lemma 2.3.2 and (3.2.3), we have the following structure of $G$ :

3.2.4 There exists a vertex set $W \subseteq V(G)$ and a partition $Y_{1}, \cdots, Y_{n}$ of $V(G)-W$, and a subset $X_{i} \subseteq Y_{i}$, for $1 \leq i \leq n$ such that

(a) $|W|+\sum_{1 \leq i \leq n}\left\lfloor\frac{1}{2}\left|X_{i}\right|\right\rfloor \leq 6$,

(b) for any $i$ with $1 \leq i \leq n$, no vertex in $Y_{i}-X_{i}$ has a neighbor in $V(G)-\left(W \cup Y_{i}\right)$ and $Y_{i} \cap\left(\cup_{j=1}^{3} L_{j}\right) \subseteq X_{i}$, and

(c) every good path $P$ in $G-W$ has an edge with both ends in $Y_{i}$ for some $i$.

We choose $X_{1}, Y_{1}, X_{2}, Y_{2}, \cdots, X_{n}, Y_{n}$ such that

(i) $|W|$ is as large as possible.

(ii) Subject to (i), $n$ is the largest number such that $Y_{i} \neq \emptyset$ for any $i \in\{1,2, \cdots, n\}$.

By the definition of $W,(3.2 .4)(\mathrm{b})$ and $(3.2 .4)(\mathrm{c})$, we have the following immediate observations:

3.2.5 $W \cup X_{1} \cup \cdots \cup X_{n} \supseteq L_{1} \cup L_{2} \cup L_{3}$, and $|W|+\sum_{i=1}^{n}\left|X_{i}\right| \geq\left|L_{1} \cup L_{2} \cup L_{3}\right|$. 
3.2.6 We claim that $n \geq 3+|W|$.

Since $\left|L_{1} \cup L_{2} \cup L_{3}\right|=15$ and $|W| \leq 6$ (by (3.2.4)(a)), by (3.2.5), we have $n \geq 1$. By $(3.2 .4)(\mathrm{a})$, and (3.2.5), we have

$12 \geq 2\left(|W|+\sum_{1 \leq i \leq n}\left\lfloor\frac{1}{2}\left|X_{i}\right|\right\rfloor\right) \geq 2|W|+\sum_{1 \leq i \leq n}\left|X_{i}\right|-n \geq|W|+\left|L_{1} \cup L_{2} \cup L_{3}\right|-n \geq|W|+15-n$.

Thus,

$$
n \geq 3+|W|
$$

3.2.7 Definition of $A_{i}($ for $i=1,2,3)$

Let $G^{\prime \prime}$ be the subgraph obtained from $G-W$ by deleting all edges contained in any $Y_{j}$. Let $A_{i}$ be the union of the vertex subsets of all components of $G^{\prime \prime}$ containing some vertex of $L_{i}$ for each $i \in\{1,2,3\}$.

\subsubsection{Properties of $\left\{A_{1}, A_{2}, A_{3}\right\}$}

Properties of $\left\{A_{1}, A_{2}, A_{3}\right\}$ are to be studied in this subsection. The first property is immediate by (3.2.4) and the definition of $A_{i}$.

(a) $L_{i}-W \subseteq A_{i} \subseteq V(G)-W$ for $i=1,2,3$.

Note that each $Y_{j}-X_{j}$ is an independent set of $G^{\prime \prime}$, and by $(3.2 .4)(\mathrm{b})$, we have the following properties.

(b) $A_{i} \subseteq X_{1} \cup \cdots \cup X_{n}$ for $i=1,2,3$.

(c) $A_{1}, A_{2}, A_{3}$ are disjoint by the definition of $A_{i}$ and $(3.2 .4)(\mathrm{c})$.

3.2.9 We claim that $|W| \leq 5$. 
For otherwise, by (3.2.4)(a), we may assume that $|W|=6$.

By (3.2.4)(a), $\left|X_{i}\right| \leq 1$ for $1 \leq i \leq n$. By the choice of $|W|$ and $n, Y_{i}$ is a connected subgraph.

For $1 \leq j \leq 3$, let $\overline{A_{j}}$ be the union of all $Y_{i} s$ if $Y_{i} \cap A_{j} \neq \emptyset$ for $1 \leq i \leq n$.

By $(3.2 .4)(\mathrm{b}),(3.2 .4)(\mathrm{c})$ and the fact that $\left|X_{i}\right| \leq 1$ for $1 \leq i \leq n, \overline{A_{j}}$ and $\overline{A_{k}}$ are disconnected in $G-W$ for $1 \leq j<k \leq 3$. Hence, $W$ is a vertex cut of $G$ separating $\overline{A_{1}}, \overline{A_{2}}$, and $\overline{A_{3}}$.

Note that $G$ is 6 -connected and $|W|=6, W$ is a minimal vertex cut of $G$.

If $\overline{A_{j}} \neq \emptyset$ for each $j \in\{1,2,3\}$, then every vertex $w$ of the minimal cut $W$ must be adjacent to at least one vertex of each $\overline{A_{j}}$. This contradicts that $G$ is claw-free.

Hence, without loss of generality, we may assume that $\overline{A_{1}}=\emptyset$.

(i) We claim that $\overline{A_{j}} \neq \emptyset$ for $j \in\{2,3\}$. For otherwise, we may assume that $\overline{A_{2}}=\emptyset$, then both $L_{1}$ and $L_{j}$ are contained in $W$. this contradicts that $L_{1}$ and $L_{j}$ are disjoint and $|W|=6$.

(ii) We claim that $W \subseteq N_{G}\left(\overline{A_{j}}\right)$ for $j \in\{2,3\}$. For otherwise, $\left|N_{G}\left(\overline{A_{j}}\right) \cap W\right|<|W|=6$. By $(3.2 .4)(\mathrm{b})$ and $(3.2 .4)(\mathrm{c}), N_{G}\left(\overline{A_{j}}\right) \cap W$ is a vertex cut separating $\overline{A_{j}}$ from $V(G)-\overline{A_{j}}-W$. It contradicts that $G$ is 6 -connected.

(iii) Since $\overline{A_{1}}=\emptyset, L_{1} \subseteq W$. By (3.2.9)(i) and (ii), we have $W \subseteq N_{G}\left(\overline{A_{j}}\right)$ for $j \in\{2,3\}$. Hence, we contract $\overline{A_{2}} \cup\left(W-L_{1}\right)$ to new vertex $u$ and contract $\overline{A_{3}}$ to new vertex $v, L_{1} \cup\{u, v\}$ is a 7 -clique. It is a contradiction.

3.2.10 We claim that $W \cup X_{i}$ is a vertex cut of $G$ if $Y_{i}-X_{i} \neq \emptyset$.

Since $n \geq 3$ by (3.2.6), $V(G)-W-Y_{i} \neq \emptyset$. Hence, by (3.2.4)(b), $W \cup X_{i}$ separates $Y_{i}-X_{i}$ from $V(G)-W-Y_{i}$.

3.2.11 We claim that $X_{i} \neq \emptyset$ for all $i$.

Without loss of generality, we assume that $X_{1}=\emptyset$. Note that $Y_{1} \neq \emptyset$. Hence, by (3.2.10), $W$ is a vertex cut of $G$, but $|W| \leq 5$ by (3.2.9), it contradicts that $G$ is 6 -connected.

3.2.12 We claim that $\left|X_{i}\right|$ is odd for all $i$. 
Suppose that $\left|X_{1}\right|$ is even, then by (3.2.11), $\left|X_{1}\right| \geq 2$. Assume $v \in X_{1}$, let $W^{*}=W \cup\{v\}$, $Y_{1}^{*}=Y_{1}-v, X_{1}^{*}=X_{1}-v$ and $X_{i}^{*}=X_{i}, Y_{i}^{*}=Y_{i}$ for $2 \leq i \leq n$. Hence, the partition $\left\{W^{*}\right.$, $\left.X_{1}^{*}, \cdots, X_{n}^{*}, Y_{1}^{*}, \cdots, Y_{n}^{*}\right\}$ of $V(G)$ satisfies (3.2.4)(a)- (c), contradicting the choice that $|W|$ is as large as possible.

3.2.13 We claim that, for $1 \leq j \leq n$, if $\left|W \cup X_{j}\right|<6$ then $X_{j}=Y_{j}$.

This is an immediate corollary of (3.2.10).

\subsubsection{Some definitions.}

(1) Without loss of generality, we assume that $\left|X_{1}\right| \geq\left|X_{2}\right| \geq \cdots \geq\left|X_{n}\right|$, and let m be the greatest integer such that $\left|X_{m}\right| \geq 3$.

(2) $B_{i}=A_{i} \cap\left(X_{1} \cup X_{2} \cup \cdots \cup X_{m}\right)$ for $1 \leq i \leq 3$.

(3) Without loss of generality, we assume that $\left|B_{1}\right| \leq\left|B_{2}\right| \leq\left|B_{3}\right|$.

3.2.15 Properties of $\left\{B_{1}, B_{2}, B_{3}\right\}$.

(a) $B_{i} \subseteq A_{i} \subseteq X_{1} \cup \cdots \cup X_{n}$ for $i=1,2,3$ by (3.2.8)(b).

(b) $B_{1}, B_{2}, B_{3}$ are disjoint by the definition of $B_{i}$ and $(3.2 .8)(\mathrm{c})$.

3.2.16 We claim that, if $A_{i}-B_{i} \neq \emptyset$, then $W \cup B_{i}$ is a vertex cut of $G$ separating $A_{i}-B_{i}$ from $V(G)-A_{i}-W$.

Without loss of generality, we assume that $A_{1}-B_{1} \neq \emptyset$, and without loss of generality, it is sufficient to show that $W \cup B_{1}$ is a vertex cut of $G$ separating $A_{1}-B_{1}$ from $V(G)-A_{1}-W$.

By the definition of $A_{i}$ (3.2.7), we have $\left|V(G)-A_{1}\right| \geq\left|A_{2} \cup A_{3} \cup W\right| \geq\left|L_{2} \cup L_{3}\right|=10$. By (3.2.9), we have $|W| \leq 5$. Therefore $\left|V(G)-A_{1}-W\right|=\left|V(G)-A_{1}\right|-|W| \geq 5$. That is, $V(G)-A_{1}-W \neq \emptyset$.

By $(3.2 .4)(\mathrm{b})$ and $(3.2 .4)(\mathrm{c}), W \cup B_{1}$ is a vertex cut separating $A_{1}-B_{1}$ from $V(G)-A_{1}-W$. 
3.2.17 We claim that, if $A_{i}-B_{i} \neq \emptyset$ and $\left|W \cup B_{i}\right|=6$, then $W \subseteq N_{G}\left(A_{i}-B_{i}\right)$ and $W \subseteq$ $N_{G}\left(V(G)-A_{i}-W\right)$.

By (3.2.16), $W \cup B_{i}$ is a vertex cut of $G$ separating $A_{i}-B_{i}$ from $V(G)-A_{i}-W$.

Note that $G$ is 6-connected and $\left|W \cup B_{i}\right|=6, W \cup B_{i}$ is a minimal cut of $G$. Hence, every vertex of $W \cup B_{i}$ is adjacent to some vertex of $A_{i}-B_{i}$, that is, $W \subseteq N_{G}\left(A_{i}-B_{i}\right)$. With the similar argument, we have $W \subseteq N_{G}\left(V(G)-A_{i}-W\right)$.

3.2.18 We claim that $\left|B_{1} \cup B_{2} \cup B_{3}\right| \leq 18-3|W|$, if equality holds then $\left|X_{1}\right|=\left|X_{2}\right|=\cdots=$ $\left|X_{m}\right|=3$ and $m=6-|W|$.

By $(3.2 .4)(\mathrm{a}),(3.2 .15)(\mathrm{a}),(3.2 .15)(\mathrm{b})$ and $(3.2 .14)(1)$, we have

$$
\left|B_{1} \cup B_{2} \cup B_{3}\right| \leq \sum_{1 \leq j \leq m}\left|X_{j}\right| \leq 3 \sum_{1 \leq j \leq m}\left\lfloor\frac{1}{2}\left|X_{j}\right|\right\rfloor=3 \sum_{1 \leq j \leq n}\left\lfloor\frac{1}{2}\left|X_{j}\right|\right\rfloor \leq 3(6-|W|) .
$$

And if equality holds then $\left|X_{1}\right|=\left|X_{2}\right|=\cdots=\left|X_{m}\right|=3$ and $m=6-|W|$.

3.2.19 We claim that $\left|B_{i}\right| \geq 5-|W|$ for $1 \leq i \leq 3$.

By (3.2.9), we have $|W| \leq 5$. It is obvious if $|W|=5$. Therefore, we may assume $|W| \leq 4$, and it is sufficient to show $\left|B_{1}\right| \geq 5-|W|$ by $(3.2 .14)(3)$.

For otherwise, if $\left|B_{1}\right| \leq 4-|W|$, then $\left|B_{1} \cup W\right| \leq 4$. By (3.2.8)(a), we have $\left|A_{1}\right| \geq\left|L_{1}-W\right| \geq$ $5-|W|$, therefore $\left|A_{1}-B_{1}\right| \geq 1$.

Therefore, by (3.2.16), $W \cup B_{1}$ is a vertex-cut. Note that $\left|B_{1} \cup W\right| \leq 4$. It contradicts the fact that $G$ is 6 -connected.

3.2.20 We claim that, if $\left|B_{1}\right|=6-|W|$ then $|W| \geq 2$. 
By (3.2.19), (3.2.14)(3) and (3.2.18), we have $\left|B_{1}\right|=\left|B_{2}\right|=\left|B_{3}\right|=6-|W|, m=6-|W|$ and $\left|X_{1}\right|=\cdots=\left|X_{m}\right|=3$.

For otherwise, we assume that $|W| \leq 1$. Then, by (3.2.13), $Y_{i}=X_{i}$ for $1 \leq i \leq n$.

(i) We claim that $\left|B_{i} \cap X_{j}\right| \leq 1$ for $1 \leq i \leq 3,1 \leq j \leq m$. For otherwise, we may assume that $\left|B_{1} \cap X_{1}\right| \geq 2$. Note that $\left(B_{1} \Delta X_{1}\right) \cup W$ separates $\left(B_{1} \cap X_{1}\right) \cup\left(A_{1}-B_{1}\right)$ from $V(G)-A_{1}-X_{1}-W$ by $(3.2 .4)(\mathrm{b})$ and $(3.2 .4)(\mathrm{c})$, and both $\left.\left(B_{1} \cap X_{1}\right) \cup\left(A_{1}-B_{1}\right)\right)$ and $V(G)-B_{1}-X_{1}$ are non-empty. Since $G$ is 6-connected, $\left|\left(B_{1} \Delta X_{1}\right) \cup W\right| \geq 6$, but $\left|\left(B_{1} \Delta X_{1}\right) \cup W\right|=\left|B_{1} \cup W\right|+\left|X_{1}\right|-2\left|B_{i} \cap X_{j}\right| \leq$ $6+3-4=5$. It is a contradiction.

(ii) Note that $m=6-|W|,\left|B_{i}\right|=6-|W|$ and $\left|B_{i} \cap X_{j}\right| \leq 1$ for $1 \leq i \leq 3,1 \leq j \leq m$ by (3.2.20)(i), we have that $\left|B_{i} \cap X_{j}\right|=1$ for $1 \leq i \leq 3,1 \leq j \leq m$.

(iii) By (3.2.20)(ii), let $\left\{x_{i j}\right\}=B_{i} \cap X_{j}$ for $1 \leq i \leq 3,1 \leq j \leq m$. We claim that $N_{G}\left(x_{i j}\right) \cap X_{j} \neq \emptyset$. For otherwise, we assume that $N_{G}\left(x_{11}\right) \cap X_{1}=\emptyset$, then let $W^{*}=W$, $Y_{1}^{*}=Y_{1}, X_{1}^{*}=X_{1}-\left\{x_{11}\right\}, X_{i}^{*}=X_{i}, Y_{i}^{*}=Y_{i}$ for $2 \leq i \leq n$ and $Y_{n+1}^{*}=X_{n+1}^{*}=\left\{x_{11}\right\}$. Hence, the partition $\left\{W^{*}, X_{1}^{*}, \cdots, X_{n}^{*}, X_{n+1}^{*}, Y_{1}^{*}, \cdots, Y_{n}^{*}, Y_{n+1}^{*}\right\}$ of $V(G)$ satisfies (3.2.4)(a)-(c), contradicting the choices of $|W|$ and $n$.

(iv) We claim that $\left|N_{G}\left(x_{i j}\right) \cap A_{i}\right| \leq 4$. For otherwise, we assume that $\left|N_{G}\left(x_{11}\right) \cap A_{1}\right| \geq 5$. Note that $N_{G}\left(x_{11}\right) \cap X_{1}$ is nonempty by (3.2.20)(iii), and there are no edges between $N_{G}\left(x_{11}\right) \cap A_{1}$ and $N_{G}\left(x_{11}\right) \cap X_{1}$. Since $G$ is claw-free, $G-W$ is claw-free. Hence, $N_{G}\left(x_{11}\right) \cap A_{1}$ is a clique. That is, $\left\{x_{11}\right\} \cup\left(N_{G}\left(x_{11}\right) \cap A_{1}\right)$ is a clique of order at least 6 . It contradicts (3.2.1).

(v) We claim that $|W| \neq 0$ and $W \subseteq N_{G}\left(x_{i j}\right)$. It is obvious since $N_{G}\left(x_{i j}\right) \subseteq W \cup A_{i} \cup\left(X_{j}-\right.$ $\left.\left\{x_{i j}\right\}\right),\left|N_{G}\left(x_{i j}\right)\right| \geq 7$ and $\left|\left(N_{G}\left(x_{i j}\right) \cap A_{i}\right) \cup\left(X_{j}-\left\{x_{i j}\right\}\right)\right| \leq 6$ by (3.2.20)(iv).

(vi) Let $\{x\}=W$, then, by $(3.2 .20)(\mathrm{v})$, we have $x x_{11}, x x_{22} x x_{33} \in E(G)$ and there are no edges between $x_{11}, x_{22}, x_{33}$ by $(3.2 .4)(\mathrm{b})$. Hence, we get a claw centered at $x$. It is a contradiction.

3.2.21 We claim that $\left|B_{1}\right|=5-|W|$.

For otherwise, by (3.2.19), (3.2.14)(3) and (3.2.18), we have $\left|B_{1}\right|=6-|W|$. Therefore, by $(3.2 .14)(3)$, and (3.2.18), $\left|B_{1}\right|=\left|B_{2}\right|=\left|B_{3}\right|=6-|W|, m=6-|W|$ and $\left|X_{1}\right|=\cdots=\left|X_{m}\right|=3$.

(i) We claim that at most one of $A_{1}-B_{1}, A_{2}-B_{2}, A_{3}-B_{3}$ is non-empty. For otherwise, we assume that $A_{2}-B_{2} \neq \emptyset$ and $A_{3}-B_{3} \neq \emptyset$, let $x_{2} \in A_{2}-B_{2}, x_{3} \in A_{3}-B_{3}$, if $A_{1}-B_{1} \neq \emptyset$, then by (3.2.17) and (3.2.20), we have a claw centered in $W$. Hence, $A_{1}=B_{1}$, that is $L_{1} \subseteq B_{1} \cup W$. Note that $|W| \geq 2$ by (3.2.20), therefore, we can assume that $x \in L_{1} \cap W$, and $x_{1} \in L_{1} \cap B_{1}$. Hence, we get a claw centered at $x$, a contradiction. 
(ii) Without loss of generality, we assume that $A_{1}-B_{1}=\emptyset$ and $A_{2}-B_{2}=\emptyset$. Therefore $\left(L_{1} \cup L_{2}\right) \subseteq W \cup B_{1} \cup B_{2}$. That is, $10 \leq 12-|W|$. Hence, $|W| \leq 2$. By (3.2.20), we have $|W|=2$. Therefore $\left|B_{1}\right|=\left|B_{2}\right|=6-|W|=4$. Therefore $\left|W \cap L_{1}\right|=\left|W \cap L_{2}\right|=1$. With the similar argument as above, we have $A_{3}-B_{3} \neq \emptyset$. By (3.2.13), $Y_{i}=X_{i}$ for $1 \leq i \leq m$. Note that $\delta(G) \geq 7$ and $\left|N_{G}(x)\right| \leq|W|+\left|A_{1}-x\right|+2=7$ for $x \in B_{1}$, hence, $W \subseteq N_{G}(x)$ for $x \in B_{1}$. Let $v \in W \cap L_{2}, x_{1} \in B_{1}$. Hence, $v x_{1} \in E(G)$. Without loss of generality, we may assume that $x_{1} \in B_{1} \cap X_{1}$. Since $\left|B_{2}\right|=4$ and $\left|X_{1}\right|=3$, we may select $x_{2} \in\left(B_{2}-X_{1}\right)$. Let $x_{3} \in A_{3}-B_{3}$, we have $v x_{3} \in E(G)$ by (3.2.17). Hence, we have a claw centered at $v$. It is a contradiction.

3.2.22 We claim that, if $\left|B_{i}\right|=5-|W|$ for some $1 \leq i \leq 3$, then $A_{i}=B_{i}$ and $L_{i}=W \cup B_{i}$.

First we show that $A_{i}=B_{i}$. For otherwise, we assume that $A_{i}-B_{i} \neq \emptyset$. By (3.2.16), $W \cup B_{i}$ is a vertex cut of $G$. Note that $\left|W \cup B_{i}\right|=5$, it contradicts the fact that $G$ is 6 -connected.

Since $L_{i} \subseteq W \cup A_{i}=W \cup B_{i}$, and $\left|W \cup B_{i}\right|=5=\left|L_{i}\right|$, we have $L_{i}=W \cup B_{i}$.

3.2.23 We claim that, if $|W| \neq 0$ then $\left|B_{2}\right|=6-|W|$.

By (3.2.21), we have $\left|B_{1}\right|=5-|W|$. By (3.2.18), (3.2.19), and (3.2.21), $\left|B_{2}\right|$ is either $6-|W|$ or $5-|W|$.

If $\left|B_{2}\right|=5-|W|$. By (3.2.22), we have $L_{2}=W \cup B_{2}$. Note that $\left|B_{1}\right|=5-|W|$ by (3.2.21), we have $L_{1}=W \cup B_{1}$ by (3.2.22). Since $L_{1}$ and $L_{2}$ are disjoint, $|W|=0$, it is a contradiction.

3.2.24 We claim that $|W| \leq 1$.

For otherwise, we may assume that $|W| \geq 2$. By (3.2.21), we have $\left|B_{1}\right|=5-|W|$. By (3.2.22), we have $A_{1}=B_{1}$ and $L_{1}=W \cup B_{1}$. By (3.2.23), we have $\left|B_{2}\right|=6-|W|$. By (3.2.18), (3.2.21), and (3.2.23), we have $6-|W| \leq\left|B_{3}\right| \leq 7-|W|$.

(i) We claim that, for $i \in\{2,3\}$, if $\left|B_{i}\right|=6-|W|$, then $A_{i}-B_{i} \neq \emptyset$ and $W \subseteq N_{G}\left(A_{i}-B_{i}\right)$. Since $|W| \geq 2$, we have $\left|B_{i}\right|=6-|W| \leq 4$, note that $\left|L_{i}\right|=5, L_{i}-B_{i} \neq \emptyset$. Therefore $A_{i}-B_{i} \neq \emptyset$. Note that $\left|W \cup B_{i}\right|=6$ and $A_{i}-B_{i} \neq \emptyset$, by (3.2.17), we have $W \subseteq N_{G}\left(A_{i}-B_{i}\right)$.

(ii) We claim that $|W| \leq 4$. For otherwise, by (3.2.9), we may assume that $|W|=5$. Note that $L_{1}=W \cup B_{1}$ and $\left|B_{1}\right|=5-5=0$, therefore $W=L_{1}$. Since $\left|B_{2}\right|=6-|W|, A_{2}-B_{2} \neq \emptyset$ and $W \subseteq N_{G}\left(A_{2}-B_{2}\right)$ by (3.2.24)(i). By (3.2.17), we have $W \subseteq N_{G}\left(V(G)-W-A_{2}\right)$. Hence, 
we contract $\left(A_{2}-B_{2}\right), B_{2} \cup\left(V(G)-W-A_{2}\right)$ into new vertices $u, v$ respectively, $L_{1} \cup\{u, v\}$ is a $K_{7}$ clique. It is a contradiction.

(iii) We claim that $\left|B_{3}\right|=7-|W|$. For otherwise, we assume that $\left|B_{3}\right|=6-|W|$. By (3.2.24)(i), for $i=2,3$, we have $A_{i}-B_{i} \neq \emptyset$ and $W \subseteq N_{G}\left(A_{i}-B_{i}\right)$. Since $\left|L_{1}\right|=5$ and $|W| \leq 4$ (by (3.2.24)(ii)), we have $L_{1}-W \neq \emptyset$. Let $x \in W, x_{1} \in\left(L_{1}-W\right)$, and $x_{2} \in\left(A_{2}-B_{2}\right) \cap N_{G}(x)$, $x_{3} \in\left(A_{3}-B_{3}\right) \cap N_{G}(x)$. By the definition of $A_{i}$ and $B_{i}$, there is no edge among $x_{1}, x_{2}, x_{3}$, we get a claw centered at $x$, it is a contradiction.

(iv) We claim that, if $\left|B_{3}\right| \leq 4$, then $A_{3}-B_{3} \neq \emptyset$ and $N_{G}\left(A_{3}-B_{3}\right) \cap W \neq \emptyset$. Since $\left|A_{3}\right| \geq\left|L_{3}\right|=5$ and $\left|B_{3}\right| \leq 4, A_{3}-B_{3} \neq \emptyset$. By (3.2.16), $W \cup B_{3}$ is a vertex cut of $G$ separating $A_{3}-B_{3}$ from $V(G)-A_{3}-W$. If $N_{G}\left(A_{3}-B_{3}\right) \cap W=\emptyset$, then $B_{3}$ is a vertex cut of $G$. Note that $\left|B_{3}\right| \leq 4$, it contradicts the fact that $G$ is 6 -connected.

(v) We claim that $|W|=2$. For otherwise, $\left|B_{3}\right|=7-|W| \leq 4$, then, by (3.2.24)(iv), $A_{3}-B_{3} \neq \emptyset, N_{G}\left(A_{3}-B_{3}\right) \cap W \neq \emptyset$. Let $x \in N_{G}\left(A_{3}-B_{3}\right) \cap W$, and $x_{1} \in L_{1}-W, x_{2} \in$ $\left(A_{2}-B_{2}\right) \cap N_{G}(x), x_{3} \in\left(A_{3}-B_{3}\right) \cap N_{G}(x)$, there is no edge among $x_{1}, x_{2}, x_{3}$ by (3.2.4)(b). Hence, we get a claw, it is a contradiction.

(vi) By (3.2.18), (3.2.24)(iii) and (3.2.24)(v), we have $m=6-|W|=4$ and $\left|X_{1}\right|=\cdots=$ $\left|X_{m}\right|=3$. Hence, by (3.2.13), we have $Y_{i}=X_{i}$ for $1 \leq i \leq n$. Let $x \in L_{1}-W$, we may assume that $x \in X_{1}$, therefore $N_{G}(x) \subseteq\left(A_{1}-\{x\}\right) \cup W \cup\left(X_{1}-L_{1}\right)=\left(B_{1}-\{x\}\right) \cup W \cup\left(X_{1}-L_{1}\right)$, that is $\left|N_{G}(x)\right| \leq(5-|W|-1)+|W|+2=4+2=6$. It contradicts that $\delta(G) \geq 7$.

3.2.25 We claim that, if $\left|X_{i}\right| \leq 3$ for some $1 \leq i \leq n$, then $X_{i}=Y_{i}$.

By (3.2.13) and (3.2.24), it is obvious.

3.2.26 We claim that, if $\left|B_{i}\right|=5-|W|$ for some $1 \leq i \leq 3$, then $\left|B_{i} \cap X_{j}\right|=0$ for each $X_{j}$ with $\left|X_{j}\right|=3$.

For otherwise, let $x \in B_{i} \cap X_{j}$. Since $\left|X_{j}\right|=3$, by (3.2.25), we have $X_{j}=Y_{j}$. Note that $\left|B_{i}\right|=5-|W|$, by (3.2.22), we have $A_{i}=B_{i}$. Hence, $N_{G}(x) \subseteq\left(X_{j}-\{x\}\right) \cup W \cup\left(B_{i}-\{x\}\right)$ by $(3.2 .4)(\mathrm{b})$ and $(3.2 .4)(\mathrm{c})$. That is, $\left|N_{G}(x)\right| \leq\left|\left(X_{j}-\{x\}\right) \cup W \cup\left(B_{i}-\{x\}\right)\right| \leq 6$. It contradicts that $\delta(G) \geq 7$.

\subsubsection{We claim that}


(i) $\quad m \geq 2|W|+\left|X_{1} \cup X_{2} \cup \cdots \cup X_{m}\right|-12 \geq 2|W|+\left|B_{1} \cup B_{2} \cup B_{3}\right|-12$;

(ii) There exists $X_{i}$ with $\left|X_{i}\right| \geq 5$ for $1 \leq i \leq m$;

(iii) $\quad m \leq 5-|W|$.

Proof of (i): By (3.2.4)(a), and (3.2.12), we have

$$
12 \geq 2\left(|W|+\sum_{1 \leq i \leq n}\left\lfloor\frac{1}{2}\left|X_{i}\right|\right\rfloor\right)=2\left(|W|+\sum_{1 \leq i \leq m}\left\lfloor\frac{1}{2}\left|X_{i}\right|\right\rfloor\right)=2|W|+\sum_{1 \leq i \leq m}\left|X_{i}\right|-m
$$

Thus,

$$
m \geq 2|W|+\left|X_{1} \cup X_{2} \cup \cdots \cup X_{m}\right|-12 .
$$

The second part of the inequality follows by (3.2.14)(1)-(3).

Proof of (ii): For otherwise, we assume that $\left|X_{i}\right|=3$ for $1 \leq i \leq m$. Note that $\left|B_{1}\right|=5-|W|$ by (3.2.21). By (3.2.26), $B_{1} \cap X_{i}=\emptyset$ for $1 \leq i \leq m$. Hence, $B_{1}=\emptyset$. It contradicts that $\left|B_{1}\right|=5-|W| \geq 4$ (by $(3.2 .21)$ and (3.2.24)).

Proof of (iii): By (3.2.27)(i) and (3.2.27)(ii), we have,

$$
m \geq 2|W|+\left|X_{1} \cup X_{2} \cup \cdots \cup X_{m}\right|-12 \geq 2|W|+(3 m+2)-12=2|W|+3 m-10 .
$$

Thus, $m \leq 5-|W|$.

\subsubsection{We claim that $|W|=0$.}

For otherwise, we may assume that $|W|=1$ by (3.2.24). By (3.2.21), we have $\left|B_{1}\right|=$ $5-|W|=4$. By (3.2.23), we have $\left|B_{2}\right|=6-|W|=5$. Hence, $5 \leq\left|B_{3}\right| \leq 6$ by (3.2.14)(3) and (3.2.18). Therefore $\left|B_{1} \cup B_{2} \cup B_{3}\right|$ is either 14 or 15 .

By $(3.2 .27)($ iii), we have $m \leq 5-|W|=4$.

By (3.2.27)(i), we have

$$
m \geq 2|W|+\left|X_{1} \cup X_{2} \cup \cdots \cup X_{m}\right|-12 \geq 2|W|+\left|B_{1} \cup B_{2} \cup B_{3}\right|-12 \geq 2+14-12=4 .
$$


Hence, $m=4$. Furthermore, the fact that all equalities hold implies that $\mid X_{1} \cup X_{2} \cup \cdots \cup$ $X_{m}|=| B_{1} \cup B_{2} \cup B_{3} \mid=14$. Therefore, the only possibility is $\left\{\left|X_{1}\right|,\left|X_{2}\right|, \cdots,\left|X_{4}\right|\right\}=\{5,3,3,3\}$. In this case, by (3.2.26) and $\left|B_{1}\right|=5-|W|$, we have $B_{1} \subseteq X_{1}$. Hence, we get a cut-set $\left(X_{1}-B_{1}\right) \cup W$ separating $B_{1} \cup\left(Y_{1}-X_{1}\right)$ from $V(G)-W-Y_{1}$. Note that $\left|\left(X_{1}-B_{1}\right) \cup W\right| \leq 2$, it is a contradiction.

\subsubsection{We claim that}

(i) $\left|B_{2}\right|=5$;

(ii) $5 \leq\left|B_{3}\right| \leq 6$.

Proof of (i): By (3.2.21), (3.2.14)(3) and (3.2.18) and (3.2.28), we have, $\left|B_{1}\right|=5,5 \leq\left|B_{2}\right| \leq$ 6 , and $\left|B_{1}\right|+\left|B_{2}\right|+\left|B_{3}\right| \leq 18$.

Assume that $\left|B_{2}\right|=6$. Thus, we have $6 \leq\left|B_{3}\right|$ (by (3.2.14)(3)). Therefore, $\left|B_{1} \cup B_{2} \cup B_{3}\right| \geq$ 17 .

By $(3.2 .27)$ (iii), and (3.2.28), we have $m \leq 5$.

By (3.2.27)(i), we have $m \geq 2|W|+\left|X_{1} \cup X_{2} \cup \cdots \cup X_{m}\right|-12 \geq 2|W|+\left|B_{1} \cup B_{2} \cup B_{3}\right|-12 \geq$ $17-12=5$.

Hence, $m=5$, and the fact that all equalities hold implies that $\left|X_{1} \cup X_{2} \cup \cdots \cup X_{m}\right|=$ $\left|B_{1} \cup B_{2} \cup B_{3}\right|=17$ and $\left|B_{3}\right|=6$. Therefore, the only possibility is $\left\{\left|X_{1}\right|,\left|X_{2}\right|, \cdots,\left|X_{5}\right|\right\}=$ $\{5,3,3,3,3\}$. By (3.2.26), we have $B_{1} \subseteq X_{1}$. By the definition of $A_{i}, B_{i}$ (see (3.2.7), (3.2.14)(ii)), the graph $A_{1} \cup X_{1}$ is not connected to other part of $G$, a contradiction.

(ii) can be proved by the similar argument as (3.2.29)(i).

3.2.30 We claim that $\left|X_{i}\right| \geq 5$ for $1 \leq i \leq m$.

For otherwise, we may assume that $\left|X_{m}\right|=3$. By (3.2.21), (3.2.29), (3.2.28) and (3.2.26), we have $B_{1} \cap X_{m}=B_{2} \cap X_{m}=\emptyset$. There are four cases:

Case 1: $X_{m} \subseteq B_{3}$. In this case, $B_{3}-X_{m}$ is a vertex-cut separating $X_{m}$ from $V(G)-B_{3}$ by (3.2.4)(b), note that $\left|B_{3}-X_{m}\right| \leq 3$, it is a contradiction that $G$ is 6-connected.

Case 2: $\left|X_{m} \cap B_{3}\right|=2$. By (3.2.29)(ii), $5 \leq\left|B_{3}\right| \leq 6$. Note that $\left|B_{3}\right| \neq 5$ by (3.2.26) and (3.2.28). Thus $\left|B_{3}\right|=6$. By (3.2.27)(i), (3.2.14)(1)-(3) and (3.2.28), note that $B_{1} \cap X_{m}=$ 
$B_{2} \cap X_{m}=\emptyset$, we have $m \geq 2|W|+\left|X_{1} \cup X_{2} \cup \cdots \cup X_{m}\right|-12 \geq\left|B_{1} \cup B_{2} \cup B_{3}\right|+\mid X_{m}-$ $B_{3} \mid=16+1-12=5$. Note that $m \leq 5$ by (3.2.27)(iii), hence, $m=5$. Therefore, all possibilities are $\left\{\left|X_{1}\right|,\left|X_{2}\right|, \cdots,\left|X_{5}\right|\right\}=\{3,3,3,3,3\}$ and $\left\{\left|X_{1}\right|,\left|X_{2}\right|, \cdots,\left|X_{5}\right|\right\}=\{5,3,3,3,3\}$. $\left\{\left|X_{1}\right|,\left|X_{2}\right|, \cdots,\left|X_{5}\right|\right\}=\{3,3,3,3,3\}$ is impossible by $(3.2 .26)$ and $\left|B_{1}\right|=5-|W|>0$. Next we focus on $\left\{\left|X_{1}\right|,\left|X_{2}\right|, \cdots,\left|X_{5}\right|\right\}=\{5,3,3,3,3\}$, in this subcase, by $(3.2 .26)$ and $\left|B_{1}\right|=\left|B_{2}\right|=5$, we have $B_{1} \subseteq X_{1}$, and $B_{2} \subseteq X_{1}$. Note that $B_{1}$ and $B_{2}$ are disjoint, hence, $B_{1} \cup B_{2} \subseteq X_{1}$ and $\left|B_{1} \cup B_{2}\right|=10$, it is a contradiction.

Case 3: $\left|X_{m} \cap B_{3}\right|=1$. By (3.2.29)(ii), $5 \leq\left|B_{3}\right| \leq 6$. Note that $\left|B_{3}\right| \neq 5$ by (3.2.26) and (3.2.28). Thus $\left|B_{3}\right|=6$. By (3.2.27)(i), (3.2.14)(1)-(3) and (3.2.28), note that $B_{1} \cap X_{m}=B_{2} \cap$ $X_{m}=\emptyset$, we have $m \geq 2|W|+\left|X_{1} \cup X_{2} \cup \cdots \cup X_{m}\right|-12 \geq\left|B_{1} \cup B_{2} \cup B_{3}\right|+\left|X_{m}-B_{3}\right|=16+2-12=6$. Note that $m \leq 5$ by $(3.2 .27)$ (iii), it is a contradiction.

Case 4: $X_{m} \cap B_{3}=\emptyset$. By (3.2.27)(i), (3.2.14)(1)-(3) and (3.2.28), note that $B_{1} \cap X_{m}=$ $B_{2} \cap X_{m}=\emptyset$, we have $m \geq 2|W|+\left|X_{1} \cup X_{2} \cup \cdots \cup X_{m}\right|-12 \geq\left|B_{1} \cup B_{2} \cup B_{3}\right|+\left|X_{m}\right| \geq 15+3-12=6$. Note that $m \leq 5$ by $(3.2 .27)$ (iii), it is a contradiction.

3.2.31 We claim that $m=3,\left|X_{1} \cup X_{2} \cup \cdots \cup X_{m}\right|=15,\left|B_{3}\right|=5$.

By $(3.2 .30),(3.2 .27)(\mathrm{i})$, and (3.2.28), we have

$$
m \geq 2|W|+\left|X_{1} \cup X_{2} \cup \cdots \cup X_{m}\right|-12 \geq 5 m-12 .
$$

Thus $m \leq 3$.

By (3.2.21), and (3.2.14)(3), and (3.2.28), we have $\left|B_{1} \cup B_{2} \cup B_{3}\right| \geq 15-3|W|=15$. Therefore by (3.2.27)(i) and (3.2.28), we have $m \geq 2|W|+\left|X_{1} \cup X_{2} \cup \cdots \cup X_{m}\right|-12 \geq 2|W|+$ $\left|B_{1} \cup B_{2} \cup B_{3}\right|-12 \geq 15-12=3$.

Hence, we have $m=3$, and the fact that all equalities hold implies that $\left|X_{1} \cup X_{2} \cup \cdots \cup X_{m}\right|=$ $\left|B_{1} \cup B_{2} \cup B_{3}\right|=15$. and $\left|B_{3}\right|=5$.

\subsubsection{The final step.}

By (3.2.31), we have $m=3$. By (3.2.30) and (3.2.31), the only possibility is $\left\{\left|X_{1}\right|,\left|X_{2}\right|,\left|X_{3}\right|\right\}=$ $\{5,5,5\}$. 
By (3.2.21), (3.2.29)(i) and (3.2.31), we have $\left|B_{i}\right|=5$ for $1 \leq i \leq 3$. By $(3.2 .28),|W|=0$. Hence, by (3.2.22), $A_{i}=B_{i}=L_{i}$. By (3.2.13), $Y_{i}=X_{i}$ for $1 \leq i \leq 3$. Thus $V(G)=$ $L_{1} \cup L_{2} \cup L_{3}=X_{1} \cup X_{2} \cup X_{3}=B_{1} \cup B_{2} \cup B_{3}$.

(i) We claim that $\left|B_{i} \cap X_{j}\right| \leq 2$ for $1 \leq i, j \leq 3$. For otherwise, we may assume that $\left|B_{1} \cap X_{1}\right| \geq 3$. Let $x \in B_{1} \cap X_{1}$, by (3.2.4)(b), $N_{G}(x) \subseteq\left(B_{1}-\{x\}\right) \cup\left(X_{1}-B_{1}\right)$. That is, $\left|N_{G}(x)\right| \leq\left|\left(B_{1}-\{x\}\right) \cup\left(X_{1}-B_{1}\right)\right| \leq 4+2=6$. It contradicts $\delta(G) \geq 7$.

(ii) Note that $\left|X_{i}\right|=5$, by (3.2.32)(i), $\left|B_{i} \cap X_{j}\right| \geq 1$ and $\left|X_{j}-B_{i}\right| \geq 3$ for $1 \leq i, j \leq 3$.

(iii) We claim that, if $x \in B_{i} \cap X_{j}$ for $1 \leq i, j \leq 3$, then $\left|N_{\left(X_{j}-B_{i}\right)}(x)\right| \geq 3$. By (3.2.4)(b) and the definition of $B_{i}((3.2 .14)(2))$, we have $N_{G}(x) \subseteq\left(B_{i}-\{x\}\right) \cup N_{\left(X_{j}-B_{i}\right)}(x) \subseteq\left(B_{i}-\{x\}\right) \cup$ $\left(X_{j}-B_{i}\right)$, that is, $\left|N_{G}(x)\right| \leq\left|B_{i}-\{x\}\right|+\left|N_{\left(X_{j}-B_{i}\right)}(x)\right|=4+\left|N_{\left(X_{j}-B_{i}\right)}(x)\right|$. Note that $\delta(G) \geq 7$, hence, we have $\left|N_{\left(X_{j}-B_{i}\right)}(x)\right| \geq 3$.

(iv) We claim that $X_{i}$ induces a clique for $1 \leq i \leq 3$. If $x, y \in X_{i}$ but $x y \notin E(G)$. Without loss of generality, let $x \in B_{1}, y \in B_{2}$. Next, we show that $\left|X_{i}-B_{1}\right| \geq 4$, for otherwise, assume $\left|X_{i}-B_{1}\right| \leq 3$. Hence, by (3.2.32)(ii) and (3.2.32)(iii), we have $\left|X_{i}-B_{1}\right|=3$ and $\left|N_{\left(X_{i}-B_{1}\right)}(x)\right|=3$. Note that $y \in B_{2} \cap X_{i}, x$ is adjacent to $y$, it is a contradiction. Symmetrically, we have $\left|X_{i}-B_{2}\right| \geq 4$. Those together imply that $\left|B_{3} \cap X_{i}\right| \geq 3$. This contradicts (3.2.32)(i).

Hence, we contract $L_{2}, L_{3}$ to two new vertices $u, v$ respectively, then $L_{1} \cup\{u, v\}$ is a 7-clique, a contradiction.

This completes the proof of this theorem. 


\section{Chapter 4}

\section{Chords of Longest Circuits in Locally Planar Graphs}

\subsection{Introduction}

An edge $e$ is called a chord of a circuit if $e$ is not an edge of the circuit and both endvertices of $e$ are in the circuit.

The following conjecture was proposed by Thomassen.

Conjecture 4.1.1 (Thomassen [1], p. 466) Every longest circuit of a 3-connected graph must have a chord.

Conjecture 4.1.1 has been verified for following families of graphs.

1. (C.-Q. Zhang [39]) 3-connected cubic planar graphs and 3-connected planar graphs with minimum degree at least four.

2. (C. Thomassen [33]) 3-connected cubic graphs 
3. (X. Li and C.-Q. Zhang [18]) 3-connected projective graphs with minimum degree at least four

4. (X. Li and C.-Q. Zhang [19]) 4-connected graphs embedded in the torus of the Klein bottle.

etc.

In this chapter, we will verify the conjecture for locally planar graphs.

Theorem 4.1.2 There is a function $h: N \longrightarrow N$, such that, for every 4-connected graph $G$ with minimum degree at least five embedded in a surface with Euler genus $g$ and face-width at least $h(g)$, every longest circuit of the graph $G$ has a chord.

\subsection{Terminology and notations}

All graphs considered in this paper are finite, undirected, and without loops or multiple edges. For a simple graph $G$, the vertex set and edge set of $G$ are denoted by $V(G)$ and $E(G)$ respectively.

Let $u, v \in V(G)$. The vertex $u$ is a neighbor of $v$ if $u v \in E(G)$. The set of all neighbors of $v$ is denoted by $N(v)$. The degree of a vertex $v$, denoted by $d(v)$, is the number of neighbors of $v$.

A surface $S$ is a compact arc-wise connected Hausdorff topological space which is locally homeomorphic to a disc (which means that, for every point $p$ on $S$, there is an open set in $S$ containing $p$ which is homeomorphic to an open disc in the plane). A surface can be obtained in the following way: Take a collection of pairwise disjoint convex polygons (and their interior) of side length 1 in the plane. Identify each side with precisely one other side (possibly in the same polygon). This results in a topological space $S$ and a simple graph $G$ (whose nodes are the corners of the polygons and whose edges are the sides).

If $G$ is connected, then also $S$ is arc-wise connected. If, in addition, $S$ is locally homeomorphic to a disc at every node of $G$, then $S$ is a surface. We say that $G$ is a 2-cell embedding of $S$.

If all the polygons are triangles(and no side of a triangle is identified with a side of the same triangle) then $G$ is triangulation of $S$ and $S$ is a triangulated surface. The simplest 
triangulated surface is the (boundary of the) tetrahedron. A face of a surface with a 2-cell embedding is the interior of one of the polygons.

We shall now define the (triangulated) surfaces $S_{g}$ and $N_{h}$.

$S_{0}$ is the sphere. If we cut out two disjoint discs in $S_{0}$ and identify their boundaries such that the clockwise orientations of these boundaries disagree, then we have added a handle to $S_{0}$. If we add $g$ handles we obtain $S_{g}$. If instead we cut out an open disc in $S_{0}$ and identify any two opposite points on the boundary, then we have added a crosscap to $S_{0}$. If we add $h$ $\operatorname{crosscaps}(h \geq 1)$, then we obtain $N_{h} . S_{1}, N_{1}, N_{2}$ are the torus, the projective plane and the Klein bottle, respectively. It is easy to see that all surfaces $S_{g}$ and $N_{h}$ are triangulated. (They can be obtained from the boundary of the tetrahedron by cutting out triangles instead of discs).

The surfaces $S_{g}$ are called orientable while the surfaces $N_{h}$ are called nonorientable.

All embedding of graphs in surfaces considered in this paper are 2-cell embedding. If $G$ is embedded in a surface $S$ with $f$ faces, then the number $g=2-|V(G)|+|E(G)|-f$ is called the Euler genus of $S$. The edge-width $\mathbf{e w}(\mathbf{G})$ of a graph $G$ embedded in a non-simply connected surface is defined as the length of a shortest non-contractible circuit in $G$. The face-width or representativeness, denoted by $\mathbf{f w}(\mathbf{G})$, is the minimum $k$ such that every non-contractible simple closed curve on the surface intersects $G$ in at least $k$ points.

\subsection{Lemmas}

The following is the key lemma for the proof of the main theorem of this chapter.

Lemma 4.3.1 (T. Böhme, B. Mohar and C. Thomassen [6]) There is a function $f: N \times R^{+} \rightarrow$ $N$ such that for every $\epsilon>0$ and every 4-connected graph $G$ of order $n$ embedded in a surface with Euler genus $g$ and face-width at least $f(g, \epsilon), G$ contains a circuit $C$ with $|V(C)|>(1-\epsilon) n$.

Lemma 4.3.2 Let $G$ be a connected graph with $|V(G)| \geq 3$ embedded in a surface $S$ with Euler genus $g$, then $|E(G)|<3|V(G)|+3 g$. 
Proof. Assume $G$ is embedded in $S$ with $f$ faces. Since each face is bounded by at least 3 edges, $3 f \leq 2|E(G)|$. By Euler formula,

$$
2-g=|V(G)|-|E(G)|+f \leq|V(G)|-|E(G)|+\frac{2|E(G)|}{3} .
$$

That is,

$$
2-g \leq|V(G)|-\frac{|E(G)|}{3}
$$

Hence,

$$
|E(G)| \leq 3|V(G)|-6+3 g<3|V(G)|+3 g
$$

\subsection{Proof of the main theorem}

The main theorem (Theorem 4.1.2) is to be proved in this section.

Choose $\epsilon \leq \frac{1}{32}$. By Lemma 4.3.1, there is a function $f: N \times R^{+} \rightarrow N$ such that, for every 4-connected graph $G$ embedded in a surface with Euler genus $g$ and face-width at least $f(g, \epsilon)$, every longest circuit of $G$ is of length at least $(1-\epsilon)|V(G)| \geq \frac{31}{32}|V(G)|$.

Let $h(g)=\max \{f(g, \epsilon), 4 g\}$. And let $G$ be a 4-connected graph with minimum degree at least five embedded in a surface with Euler genus $g$ and face-width at least $h(g)$. We will verify the conjecture for the graph $G$ by way of contradiction.

Let $C$ be a longest circuit of $G$ without chord and let $W=V(G)-V(C)$, and let $A E(C)=$ $\{u v \in E(G) \mid u v \notin E(C)$ and $\{u, v\} \cap V(C) \neq \emptyset\}$. It is obvious that

$$
|A E(C)|=\sum_{v \in V(C)}(d(v)-2)
$$

since $C$ is chordless. Furthermore,

$$
|A E(C)| \leq|E(G)-E(C)| \leq \sum_{v \in W} d(v) .
$$


That is,

$$
\sum_{v \in V(C)}(d(v)-2) \leq \sum_{v \in W} d(v) .
$$

Hence,

$$
\sum_{v \in V(C)}(2 d(v)-2) \leq \sum_{v \in V(G)} d(v) .
$$

So,

$$
\sum_{v \in V(C)}(d(v)-1) \leq E(G) .
$$

Since the minimum degree $G$ is at least 5 . we have that

$$
4|V(C)| \leq E(G)
$$

Therefore, by Lemma 4.3.2,

$$
4|V(C)| \leq E(G)<3|V(G)|+3 g .
$$

Since the face-width of $G$ is at least $h(g) \geq 4 g$, we have that $|V(G)| \geq 4 g$. Therefore,

$$
4|V(C)|<3|V(G)|+\frac{3|V(G)|}{4}=\frac{15|V(G)|}{4} .
$$

That is,

$$
|V(C)|<\frac{15|V(G)|}{16}
$$

Note that $C$ is a longest circuit of $G$. By Lemma 4.3.1, $|V(C)|>(1-\epsilon)|V(G)| \geq \frac{31|V(G)|}{32}$. This is a contradiction. 
52 CHAPTER 4. CHORDS OF LONGEST CIRCUITS IN LOCALLY PLANAR GRAPHS 


\section{Bibliography}

[1] B. Alspach and C. Godsil (Eds), Cycle in graphs, Ann. Discrete Math. 27 (1985)

[2] K. Appel and W. Haken, Every planar map is four colorable, Part I. Discharging. Illinois J. Math. 21 (1977), 429-490.

[3] K. Appel, W. Haken and J. Koch, Every planar map is four colorable, Part II. Reducibility. Illinois J. Math. 21 (1977), 491-567.

[4] L. W. Beineke, Derived graphs and digraphs, Beiträge zur Graphentheorie, Teubner, Leipzig, 1968.

[5] L. W. Beineke, Characterizations of derived graphs. J. Combin. Theory Ser. B., 9 (1970) 129-135.

[6] T. Böhme, B. Mohar and C. Thomassen, Long cycles in graphs on fixed surface. J. Combin. Theory Ser. B., 85 (2002), 338-347.

[7] G. Chartrand, D. P. Geller and T. Hedetniemi, Graphs with forbidden subgraphs. J. Combin. Theory 10 (1971), 12-41.

[8] Reinhard Diestel, Graph Theory-Second Edition, (C)2000, 1997 Springer-Verlag New York, Inc. page 79-80.

[9] G. A. Dirac, A property of 4-chromatic graphs and some remarks on critical graphs. J. London Math. Soc. 27 (1952), 85-92.

[10] H. Hadwiger, Über eine Klassifikation der Streckenkomplexe. Vierteljahrsschr. Naturforsch. Ges. Zürich 88 (1943), 133-142.

[11] R. Halin, Über einen Satz von K. Wagner zum Vierfarbenproblem. Math. Ann., 153 (1964) 47-62.

[12] F. Harary, Graph Theory, Edison-Wesley Publishing Company, Reading, (1969). 
[13] L. K. Jørgensen, Contractions to K. J. Graph Theory 18 (1994), 431-448.

[14] K. Kawarabayashi and B. Toft, Any 7-chromatic graph has $K_{7}$ or $K_{4,4}$ as a minor. (preprint.)

[15] K. Kawarabayashi, R. Luo, J. Niu, and C.-Q. Zhang, On structure of $k$-connected graphs without $K_{k}$-minor. (preprint.)

[16] K. Kawarabayashi, Minors in 7-chromatic graphs. (preprint.)

[17] A. V. Kostochka, The minimum Hadwiger number of graphs by their average degree. Metody Diskret. Analiz. 38 (1982), 37-58 [in Russian].

[18] X. Li and C.-Q. Zhang, Chords of longest circuit in 3-connected graphs. Discrete Math., (2003) (to appear).

[19] X. Li and C.-Q. Zhang, Chords of longest circuit of graphs embedded in torus and Klein bottle. J. Graph Theory, (2003) (to appear).

[20] W. Mader, Homomorphieeigenschaften und mittlere Kantendichte von Graphen. Math Ann. 174 (1967), 265-268.

[21] W. Mader, Homomorphiesätze für Graphen. Math. Ann. 178 (1968), 154-168.

[22] W. Mader, Über die Maximalzahl kreuzungsfreier H-Wege. Arch. Math. 31 (1978), 387-402.

[23] N. Robertson, unpublished notes. (privated communication.)

[24] N. Robertson, D. P. Sanders, P. D. Seymour and R. Thomas, The four-color theorem. J. Combin. Theory Ser. B 70 (1997), 2-44.

[25] N. Robertson and P. D. Seymour, Graph Minors XVI. Excluding a nonplanar graph. (preprint.)

[26] N. Robertson and P. D. Seymour, Graph Minors XX. Wagner's Conjecture. (preprint.)

[27] N. Robertson, P. D. Seymour and R. Thomas, Hadwiger conjecture for $K_{6}$-free graphs. Combinatorica 13 (1993), 279-361.

[28] P. D. Seymour, Disjoint paths in graphs. Combinatorica 29 (1980), 293-309.

[29] Z. Song and R. Thomas, The extremal function for $K_{9}$-minor. (preprint.)

[30] A. Thomason, An extremal function for contractions of graphs. Math. Proc. Cambridge Philos. Soc. 95 (1984), 261-265.

[31] A. Thomason, The extremal function for complete minors. J. Combin. Theory Ser. B 81 (2001), 318-338.

[32] C. Thomassen, 2-linked graphs. Europ. J. Combinatorics 1 (1980), 371-378. 
[33] C. Thomassen, Chords of longest circuit in cubic graphs. J. Combin. Theory Ser. B., 71 (1997), 215-230.

[34] B. Toft, On separating sets of edges in contraction-critical graphs. Math Ann. 196 (1972), 129-147.

[35] K. Wagner, Über eine Eigenschaft der ebenen Komplexe. Math. Ann. 114 (1937), 570-590.

[36] K. Wagner, Beweis einer Abschwächung der Hadwiger-Vermutung. Math Ann. 153 (1964), 26-28.

[37] D. R. Woodall, Improper colourings of graphs. In: Graph Colourings (ed. R. Nelson and R. J. Wilson), Pitman Research Notes 218, Longman 1990, 45-63.

[38] C.-Q. Zhang, Integer flows and cycle covers of graphs, Marcel Dekker Inc., New York, 1997.

[39] C.-Q. Zhang, Longest cycles and their chords. J. Graph Theory, 11 (1987), 521-529. 